\title{
Understanding and describing the liquid-crystalline states of polypeptide solutions: a coarse-grained model of PBLG in DMF
}

\author{
Liang Wu, Erich A. Müller, and George Jackson * \\ Department of Chemical Engineering, Imperial College London, South Kensington Campus, \\ London, SW7 2AZ, United Kingdom \\ E-mail: g.jackson@imperial.ac.uk
}

\begin{abstract}
A perturbation theory is employed to construct a free-energy functional capable of describing the isotropic and nematic phases of attractive rod-like particles. An algebraic van der Waals-Onsager equation of state is then developed to determine the global phase behaviour of prolate particles for various aspect ratios and strengths of the attractive potential. Compared with the phase diagram of their athermal analogues, the incorporation of an attractive potential is seen to stabilize the nematic state leading to an increase in the degree of orientational order of the system at high densities. The most salient feature of these fluids is the existence of regions of nematic-nematic phase separation. The simple model is used to describe the phase behaviour of solutions of a relatively rigid polypeptide, poly-( $\gamma$-benzyl-L-glutamate) (PBLG), in dimethylformamide (DMF); this system exhibits a unique phase separation between two liquidcrystalline states. The coarse-grained representation of the mesogen employed herein
\end{abstract}

\footnotetext{
*To whom correspondence should be addressed
} 
is a hard spherocylinder decorated with an attractive square-well potential which acts at the center of mass of the particle. A quantitative description of the experimental isotropic-nematic phase behaviour of the PBLG solutions can be achieved from the proposed model with the use of just two temperature-dependent parameters.

\section{Introduction}

Liquid crystals (LCs) ${ }^{1-3}$ are substances that exhibit phases intermediate between the crystal and liquid state. Since the first discovery of this fascinated state of matter by Reinitzer in the late nineteenth century, ${ }^{4}$ LCs have attracted interest because of the uniqueness of their thermodynamic, structural, optical, and electronic properties. Today, one can recognize LC behaviour in an ever increasing number of scenarios: apart from the common examples of solutions of soaps, detergents, and surfactants, ${ }^{2}$ the existence of lyotropic liquid-crystalline order in biomacromolecular systems is ubiquitous in nature including the phase behaviour exhibited by DNA, ${ }^{5,6}$ by stiff polymers such as polysaccharides, ${ }^{7}$ cellulose ${ }^{8,9}$ and protein fibers, ${ }^{10,11}$ and by rod-like viruses such as the tobacco mosaic virus, ${ }^{12,13}$ and the $f d$ virus. ${ }^{14-19}$ The supramolecular $\alpha$-helices formed by the self-assembly of polypeptides in solution are also found to give rise to a rich variety of mesogenic behaviour. ${ }^{20-40}$ In the current paper we seek to understand and describe the LC ordering exhibited by solutions of the polypeptide poly- $(\gamma$ -benzyl-L-glutamate) (PBLG) in dimethylformamide (DMF) which is characterized by large regions of stable nematic order and an intriguing phase coexistence between two liquidcrystalline states with markedly different concentrations of polypeptides. ${ }^{22,23,27,28,33,36,39}$

An essential requirement for the stabilization of a LC phase is that the molecules be highly anisotropic in shape, such as long rigid rods or thin flat discs. For this reason, rodlike ${ }^{41-47}$ particles are often employed as prototype models for prolate LC molecules (see reference 48 for a relatively recent review). Hard non-spherical particles are often taken as 'zeroth-order' models for lyotropic or colloidal LCs in which the appearance of anisotropic

phases is controlled by the solute concentration. ${ }^{49,50}$ Impressively, the theoretical treatment 
of hard non-spherical fluids dates back to the 1940s: in his pioneering work on nematic LCs, Onsager ${ }^{51,52}$ proposed the now well-accepted free-energy functional for the nematic state and demonstrated that when only athermal (excluded-volume) contributions are taken into account, the isotropic-nematic phase transition can be driven by entropy alone. Onsager's original second-order virial theory was extended by Parsons ${ }^{53}$ and by Lee ${ }^{54,55}$ (PL) to incorporate the contributions from the higher-order terms based on those of a reference hard-sphere system. ${ }^{56}$ Other formal approaches, such as fundamental measure theory ${ }^{57,58}$ and integral equation theory, ${ }^{59,60}$ both of which have a sound basis in statistical mechanics, also offer promising routes to the development of an accurate description of the phase behaviour and structure of hard-anisotropic particles. However, these theories involve a complicated mathematical treatment which make them difficult to apply to realistic LC models. The more commonly employed mean-field approaches for anisotropic phases such as the MaierSaupe ${ }^{61,62}$ and the related molecular-field theories ${ }^{63-67}$ (which are based the assumption that orientationally dependent attractive interactions are the molecular feature that give rise to liquid-crystalline order) are not appropriate for the description of lyotropic systems such as solutions of PBLG. This is because the LC phases exhibited by these polypeptides are governed principally by the repulsive interactions between the highly anisometric rod-like macromolecules. The neglect of anisotropy in the shape of the molecules (which give rise to anisotropic repulsive interactions) in Maier-Saupe and molecular-field theories means that an Onsager-like treatment is therefore preferable in our case, highlighting the role played by the repulsive (excluded-volume) interactions.

Having acknowledged Onsager's broadly accepted view that repulsive excluded-volume interactions are largely responsible for the formation of orientationally ordered phases, it is also important to recognize that temperature often plays a key role in determining the relative stability of LC phases, so that attractive inter-molecular interactions have to be considered on an equal footing for a proper description of the temperature dependence of the thermodynamic properties. This is consistent with the van der Waalsian picture of fluids where the 
hard molecular core is treated as a reference which determines the fluid structure, while the dispersions (attractions) are incorporated as a perturbation. ${ }^{68-70} \mathrm{~A}$ simple hard-core model which also includes attractive interactions would certainly be appropriate to investigate the phase diagrams of thermotropic LCs. A number of studies have been carried out after the early attempts by Kimura ${ }^{71}$ to describe the properties of a system which combines Onsager's hard-rod model with anisotropic dispersion forces. ${ }^{72-83}$ Some progress has also been made in introducing dipolar ${ }^{84-89}$ or chiral ${ }^{90-93}$ interactions between LC molecules. Of particular relevance to our current work is the closed-form algebraic equation of state developed within a van der Waals-Onsager treatment for systems of attractive hard-core particles, ${ }^{94}$ in which the attractive potential is expanded in spherical harmonics ${ }^{95}$ representing different multipolar contributions to the anisotropic attractions.

A rigorous theoretical treatment of liquid-crystalline polymers is of course complicated by the fact that an individual macromolecule of this type will have additional degrees of freedom and self interactions. Nevertheless, several approaches for chain-like LC polymers based on Onsager's original theory have been developed to improve on the rigid-rod model. Khokhlov and Semenov $(\mathrm{KS})^{96}$ introduced the effect of chain flexibility as a correction to the orientational free energy of hard rods. In the KS flexible chain model, the stiffness of a chain is described with an adjustable parameter, the persistence length, conceptually defined as the characteristic length over which correlations in the direction of the chain segments are lost: for a completely flexible chain the persistence length is much smaller than the contour length which is the length at maximum physical extension of the polymer molecule. On the other hand, if the persistence length is larger than the contour length, the polymer chain can be approximated as a rigid rod-like molecule (in this case the persistence length would approach infinity). In a study which is of direct relevance to our current work, Hentschke ${ }^{97}$ showed that the KS approach can be used to describe the equation of state (osmotic pressure) of solutions of PBLG in DMF with reasonable accuracy. Though the KS flexible chains can provide a good representation of real LC polymer chains, it is 
rather difficult to obtain a general analytical expression for thermodynamic properties and orientational order due to the integro-differential equation that has to be solved to minimize the free energy of the system. ${ }^{98-101}$ A number of studies have now been made for similar flexible-chain models ${ }^{99,100,102-111}$ all of them sharing one common feature: the hard-core backbone of the polymer chains. The major drawback in implementing approaches of this type to represent real LC polymers is the strong coupling of the physical quantities in the model and the dependence of the model parameters on external fields. The persistence length, e.g., typically depends both on the model parameters (the length of a chain segment and bending energy) and on the external environment (the temperature, density, and degree of orientational order).

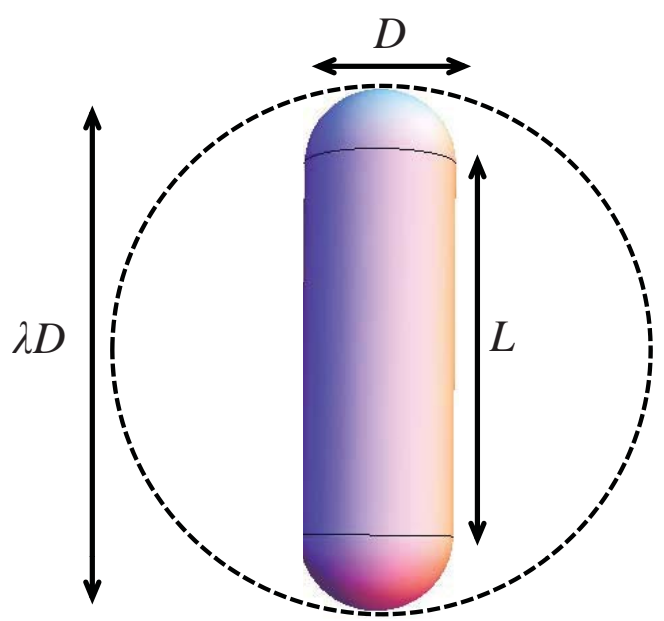

Figure 1: Square-well attractive hard spherocylinder (AHSC). The model is characterized by the length $L$ of the central cylindrical core and diameter $D$ of the hard-hemispherical caps. The dashed curve represents the attractive region bounded by the range $\lambda D$.

The fact the liquid-crystalline phases of the solutions of PBLG in DMF are found to be very sensitive to both concentration (lyotropic) and temperature (thermotropic) suggests that both repulsive and attractive interactions between the particles in the fluid should be taken into account. Here, we use an algebraic description to investigate the thermodynamics, orientational order, and fluid-phase behaviour of hard spherocylinders decorated with an anisotropic square-well (ASW) potential representing the effective inter-particle attrac- 
tive interactions. The equation of state is expressed in a closed algebraic form which allows one to add the contributions from other intermolecular interactions (e.g., chiral, dipolar, and associating interactions) in a straight-forward manner, or to extend the treatment to other types of inter-molecular potentials (e.g., Lennard-Jones or Yukawa). The effects of the anisotropy of the particle and the strength of the attraction on the phase behaviour of attractive rod-like particles are examined in detail. The attractive hard-spherocylinder (AHSC) model is depicted in Figure 1. The model is characterized by the aspect ratio $L / D$, where $L$ is the length of the cylindrical core and $D$ is the diameter, and the dimensionless parameter $\lambda$ is introduced to describe the range of the attractive interaction. We first describe the important details of the theoretical description (Section 2) and analyze the effect of the molecular parameters on the phase behaviour of model systems (Section 3). The AHSC model is then used to provide a quantitative representation of the orientational order and phase behaviour of solutions of PBLG in DMF over broad ranges of composition and temperature in Section 4. Our description of the complex PBLG macromolecule (in DMF solution) is but a coarse-grained representation of the real interactions active in the system. It is well known that the effective interaction at this level of coarse graining essentially represents a free energy (potential of mean force), and as a result the parameters characterizing the model will necessarily be temperature dependent (as seen, e.g., with the Flory $\chi$ parameter). The model is used to gain an understanding of the underlying behaviour and to provide some insight as to the nature of the effective molecular elongation and the solvent mediated interaction in different regions of the phase diagram. In order to provide a more predictive representation one would not only have to use a more realistic description of the macromolecule (determining the specific anisotropic attractive interaction from details of the molecular structure, as described, e.g., by Emelyanenko and co-workers ${ }^{112,113}$ ) but also consider the solvent molecules explicitly. 


\section{Theory}

\subsection{Generalised van der Waals-Onsager free-energy functional}

We briefly describe a first-order thermodynamic perturbation approach ${ }^{70,114}$ to derive the free energy for the nematic and isotropic phases of non-spherical attractive particles. A system of $N$ particles in a volume $V$ at a temperature $T$ is considered. The total Helmholtz free energy $A$ is represented by two contributions, a reference term (hard-core repulsive) $A^{\text {ref }}$ and a perturbation (attractive) term $A^{\text {att: }}$

$$
\frac{A}{N k T}=\frac{A^{\mathrm{ref}}}{N k T}+\frac{A^{\mathrm{att}}}{N k T},
$$

where $k$ is the Boltzmann constant. The reference term $A^{\text {ref }}$ is the free energy of a fluid of hard-body particles and therefore represents a purely repulsive contribution. Following the Parsons-Lee (PL) approach, ${ }^{53-55}$ the spatial dependence of the excluded volume is decoupled from its orientational dependence, and the reference repulsive term can then be expressed $\operatorname{as}^{94}$

$$
\begin{aligned}
\frac{A^{\mathrm{ref}}}{N k T} & =\frac{A_{\text {iso }}^{\mathrm{id}}}{N k T}+\int f(\vec{\omega}) \ln \{4 \pi f(\vec{\omega})\} \mathrm{d} \vec{\omega} \\
& +G(\eta) \iint V_{\text {exc }}\left(\vec{\omega}_{1}, \vec{\omega}_{2}\right) f\left(\vec{\omega}_{1}\right) f\left(\vec{\omega}_{2}\right) \mathrm{d} \vec{\omega}_{1} \mathrm{~d} \vec{\omega}_{2} .
\end{aligned}
$$

Here, $A_{\mathrm{iso}}^{\mathrm{id}}$ is the ideal contribution to the free energy of the isotropic phase (which incorporates all of the kinetic terms), $\vec{\omega}$ is the orientation vector of the particle, and $f(\vec{\omega})$ is the single-particle orientational distribution function. In the PL approach the density-dependent function $G(\eta)=\left(4 \eta-3 \eta^{2}\right) /\left[8(1-\eta)^{2}\right]$ represents the residual free energy of an equivalent hard-sphere system (with the same molecular volume $V_{\mathrm{m}}$ ), while $G(\eta)=\eta / 2$ in the original Onsager second-virial theory; ${ }^{48}$ in these expressions, $\eta=\rho V_{\mathrm{m}}$ is the packing fraction of the system, with $\rho=N / V$ representing the usual number density. The excluded volume $V_{\text {exc }}\left(\vec{\omega}_{1}, \vec{\omega}_{2}\right)$ corresponds to the region in phase space inaccessible to a particle with a fixed 
orientation $\vec{\omega}_{1}$ due to another particle with orientation $\vec{\omega}_{2}$, and is defined as

$$
V_{\text {exc }}\left(\vec{\omega}_{1}, \vec{\omega}_{2}\right)=\frac{1}{3} \int \sigma\left(\hat{r}_{12}, \vec{\omega}_{1}, \vec{\omega}_{2}\right) \mathrm{d} \hat{r}_{12}
$$

where $\sigma\left(\hat{r}_{12}, \vec{\omega}_{1}, \vec{\omega}_{2}\right)$ is the contact distance which depends on the relative orientations between $\vec{\omega}_{1}$ and $\vec{\omega}_{2}$ and the centre-of-mass inter-separation of the pair of anisotropic particles, $\hat{r}_{12}=\vec{r}_{12} / r_{12}$.

At the first-order level of the perturbation theory, ${ }^{94}$ the contribution due to the attractive interactions corresponds to the statistical average of attractive energy evaluated in the reference-system ensemble. Assuming that the fluid structure of the system with attractive interactions is unaltered by the perturbation and that pairwise additive interactions exist between particles, the perturbation contribution can be expressed as ${ }^{94}$

$$
\begin{aligned}
A^{\text {att }} & =\frac{1}{2} \iiint \int u_{12}^{\text {att }}\left(\vec{r}_{12}, \vec{\omega}_{1}, \vec{\omega}_{2}\right) g^{\text {ref }}\left(\vec{r}_{12}, \vec{\omega}_{1}, \vec{\omega}_{2} ; \rho\right) \\
& \times \rho^{\text {ref }}\left(\vec{r}_{1}, \vec{\omega}_{1}\right) \rho^{\text {ref }}\left(\vec{r}_{2}, \vec{\omega}_{2}\right) \mathrm{d} \vec{r}_{1} \mathrm{~d} \vec{\omega}_{1} \mathrm{~d} \vec{r}_{2} \mathrm{~d} \vec{\omega}_{2} \\
& =\frac{\rho^{2} V}{2} \iiint u_{12}^{\text {att }}\left(\vec{r}_{12}, \vec{\omega}_{1}, \vec{\omega}_{2}\right) \\
& \times f\left(\vec{\omega}_{1}\right) f\left(\vec{\omega}_{2}\right) g^{\text {ref }}\left(\vec{r}_{12}, \vec{\omega}_{1}, \vec{\omega}_{2} ; \rho\right) \mathrm{d} \vec{r}_{12} \mathrm{~d} \vec{\omega}_{1} \mathrm{~d} \vec{\omega}_{2},
\end{aligned}
$$

where $u_{12}^{\text {att }}\left(\vec{r}_{12}, \vec{\omega}_{1}, \vec{\omega}_{2}\right)$ is the attractive pair interaction potential. The pair correlation function $g^{\text {ref }}\left(\vec{r}_{12}, \vec{\omega}_{1}, \vec{\omega}_{2} ; \rho\right)$ of the reference system is a complicated function ${ }^{59,60}$ that depends on the overall density and the relative separation and orientations of the two anisotropic particles. In order to make the approach tractable, we approximate the pair distribution function of the reference system by its low-density limit, $\lim _{\rho \rightarrow 0} g^{\mathrm{ref}}\left(\vec{r}_{12}, \vec{\omega}_{1}, \vec{\omega}_{2} ; \rho\right)=1$. When considering a homogeneous nematic (anisotropic) phase, the single particle density of the system can be factorised as $\rho^{\mathrm{ref}}\left(\vec{r}_{i}, \vec{\omega}_{i}\right)=\rho f\left(\vec{\omega}_{i}\right)$, where the single particle orientation distribution function $f(\vec{\omega})$ has been introduced.

Since the particles of interest are non-spherical, the intermolecular pair potential is a function of the relative positions as well as orientations of particles. ${ }^{67,95}$ It is thus useful to expand the interaction as a series in spherical harmonics. Here we consider an anisotropic 
square-well (ASW) potential of the form: ${ }^{94,95}$

$$
\begin{aligned}
u^{\text {att }}\left(\vec{r}_{12}, \vec{\omega}_{1}, \vec{\omega}_{2}\right) & =-s\left(r_{12}\right)\left[\epsilon_{0}+\epsilon_{2} P_{2}(\cos \gamma)\right] \\
s\left(r_{12}\right) & = \begin{cases}1 & \lambda D>r_{12} \geq \sigma\left(\hat{r}_{12}, \vec{\omega}_{1}, \vec{\omega}_{2}\right) \\
0 & r_{12} \geq \lambda D\end{cases}
\end{aligned}
$$

where $\epsilon_{0}$ and $\epsilon_{2}$ characterize the isotropic and anisotropic attractive interactions respectively, $\lambda$ is the range parameter, $D$ is the reference diameter of the underlying hard-core particle, and $P_{2}(\cos \gamma)=\frac{3}{2} \cos ^{2} \gamma-\frac{1}{2}$ is the second Legendre polynomial for the relative orientation $\gamma=\arccos \left(\vec{\omega}_{1} \cdot \vec{\omega}_{2}\right)$ between the two particles. After substituting the ASW attractive potential into Equation (4) and integrating over the centre-to-centre distance between particle 1 and 2 , the perturbation free energy can be expressed as ${ }^{94}$

$$
\begin{aligned}
\frac{A^{\text {att }}}{N k T} & =-\frac{\rho \epsilon_{0}}{2 k T}\left[\frac{4 \pi}{3} \lambda^{3} D^{3}-\left\langle V_{\mathrm{exc}}(\gamma)\right\rangle_{\vec{\omega}_{1}, \vec{\omega}_{2}}\right] \\
& -\frac{\rho \epsilon_{2}}{2 k T}\left[\frac{4 \pi}{3} \lambda^{3} D^{3}\left\langle P_{2}(\cos \gamma)\right\rangle_{\vec{\omega}_{1}, \vec{\omega}_{2}}-\left\langle V_{\mathrm{exc}}(\gamma) P_{2}(\cos \gamma)\right\rangle_{\vec{\omega}_{1}, \vec{\omega}_{2}}\right] .
\end{aligned}
$$

where the the double orientational average of a quantity $J\left(\vec{\omega}_{1}, \vec{\omega}_{2}\right)$ is introduced

$$
\left\langle J\left(\vec{\omega}_{1}, \vec{\omega}_{2}\right)\right\rangle_{\vec{\omega}_{1}, \vec{\omega}_{2}}=\iint J\left(\vec{\omega}_{1}, \vec{\omega}_{2}\right) f\left(\vec{\omega}_{1}\right) f\left(\vec{\omega}_{2}\right) \mathrm{d} \vec{\omega}_{1} \mathrm{~d} \vec{\omega}_{2}
$$

Combining all of the contributions to the free energy one obtains an expression as a sum of orientational averages of the configurational terms:

$$
\begin{aligned}
\frac{A[f(\vec{\omega})]}{N k T} & =\frac{A_{\mathrm{iso}}^{\mathrm{id}}}{N k T}+\int f(\vec{\omega}) \ln [4 \pi f(\vec{\omega})] \mathrm{d} \vec{\omega}+G(\eta)\left\langle V_{\mathrm{exc}}(\gamma)\right\rangle_{\vec{\omega}_{1}, \vec{\omega}_{2}} \\
& -\frac{\rho \epsilon_{0}}{2 k T}\left[\frac{4 \pi}{3} \lambda^{3} D^{3}-\left\langle V_{\mathrm{exc}}(\gamma)\right\rangle_{\vec{\omega}_{1}, \vec{\omega}_{2}}\right] \\
& -\frac{\rho \epsilon_{2}}{2 k T}\left[\frac{4 \pi}{3} \lambda^{3} D^{3}\left\langle P_{2}(\cos \gamma)\right\rangle_{\vec{\omega}_{1}, \vec{\omega}_{2}}-\left\langle V_{\mathrm{exc}}(\gamma) P_{2}(\cos \gamma)\right\rangle_{\vec{\omega}_{1}, \vec{\omega}_{2}}\right]
\end{aligned}
$$

A clear coupling between the repulsive (excluded-volume) and attractive contributions to the pair potential can be seen in this expression: the excluded volume enters the terms 
corresponding to the isotropic and anisotropic attractive contributions, while the last term in $\left\langle V_{\text {exc }}(\gamma) P_{2}(\cos \gamma)\right\rangle_{\vec{\omega}_{1}, \vec{\omega}_{2}}$ constitutes a direct interdependence of the repulsions and attractions.

The single-particle orientational distribution function in the isotropic state is constant which simplifies the orientational integrals in Equation (8); for molecular geometries with cylindrical symmetry it corresponds to $f_{\text {iso }}(\vec{\omega})=1 / 4 \pi$. In the anisotropic state, the equilibrium orientational distribution of molecules must be found by minimizing the total freeenergy functional, $A[f(\vec{\omega})]$. In order to obtain the orientational distribution function in algebraic form, we use the Onsager trial function (OTF): ${ }^{52}$

$$
f_{\mathrm{OTF}}(\vec{\omega})=\frac{\alpha \cosh (\alpha \cos \theta)}{4 \pi \sinh (\alpha)}
$$

where the parameter $\alpha$ is introduced to characterize the degree of orientational order, the polar angle $\theta=\arccos \left(\vec{\omega} \cdot \vec{\omega}_{0}\right)$, and $\vec{\omega}_{0}$ is the director of the nematic phase. On introducing the algebraic OTF into the expression for the total free energy (8), the functional variation of $A[f(\vec{\omega})]$ with respect to $f(\vec{\omega})$ can be simplified to a derivative of the free energy with respect to the parameter $\alpha$. The simplification provided by the OTF makes the solution of the equilibrium orientational distribution straightforward. Details of the evaluations of orientational averages using the OTF have been discussed in earlier work for both rod-like (prolate) ${ }^{48,94}$ and disk-like (oblate) ${ }^{115,116}$ particles.

\subsection{Equation of state for hard spherocylinders with an anisotropic square-well potential}

The use of the OTF allows one to develop the generalized Onsager free-energy functional in algebraic form in terms of the Onsager orientational parameter $\alpha$; this facilitates the solution of the equilibrium single-particle distribution function and the isotropic-nematic coexistence. The Bessel functions involved in the orientational averages of the various contributions to the free energy can be expressed as averages of the corresponding powers of $\sin \gamma$ each of which can then be expressed algebraically in terms of $\alpha$. The choice of $\sin \gamma$ as the basis 
function for the free energy (rather than the more usual $\cos \gamma$ basis employed in spherical harmonic expansions of the potential functions) is simply a consequence of the use of the OTF approach.

For hard spherocylinders, the excluded volume of a pair of particles can be expressed $\operatorname{as}^{52}$

$$
V_{\text {exc }}(\gamma)=C_{0}+C_{1} \sin \gamma
$$

where the coefficients $C_{0}=\frac{4}{3} \pi D^{3}+2 \pi L D^{2}$ and $C_{1}=2 L^{2} D$ are determined by the shape of the particle. Similarly, the coupled term involving $V_{\text {exc }}(\gamma)$ and $P_{2}(\cos \gamma)$, or more conveniently the equivalent function $P_{2}(\sin \gamma)=1-\frac{3}{2} \sin ^{2} \gamma$, can also be written as a function of $\sin \gamma$ :

$$
V_{\text {exc }}(\gamma) P_{2}(\sin \gamma)=C_{0}+C_{1} \sin \gamma-\frac{3}{2}\left(C_{0} \sin ^{2} \gamma+C_{1} \sin ^{3} \gamma\right)
$$

The orientational averages found in the total free energy can therefore be expressed as the double orientational averages of $\left\langle\sin ^{i} \gamma\right\rangle_{\vec{\omega}_{1, \vec{\omega}_{2}}}$ where $i=0,1,2, \cdots$. The introduction of the OTF to describe the degree of orientational order of the nematic phase renders the orientational averages in algebraic form, which provides a more tractable set of expressions: ${ }^{48,94}$

$$
\begin{aligned}
\langle\sin \gamma\rangle_{\vec{\omega}_{1}, \vec{\omega}_{2}} & \approx \sqrt{\pi}\left(\frac{1}{\alpha^{1 / 2}}-\frac{15}{16 \alpha^{3 / 2}}\right)+\mathcal{O}\left(1 / \alpha^{5 / 2}\right) \\
\left\langle\sin ^{2} \gamma\right\rangle_{\vec{\omega}_{1}, \vec{\omega}_{2}} & \approx \frac{4}{\alpha}+\mathcal{O}\left(1 / \alpha^{2}\right) \\
\left\langle\sin ^{3} \gamma\right\rangle_{\vec{\omega}_{1}, \vec{\omega}_{2}} & \approx \sqrt{\pi} \frac{6}{\alpha^{3 / 2}}+\mathcal{O}\left(1 / \alpha^{5 / 2}\right) \\
\left\langle\sin ^{4} \gamma\right\rangle_{\vec{\omega}_{1}, \vec{\omega}_{2}} & \approx 0+\mathcal{O}\left(1 / \alpha^{2}\right) .
\end{aligned}
$$

Using Equations (12)-(15) and the expressions for excluded volume and second Legendre polynomial, one obtains an algebraic equation of state for hard spherocylinders interacting with an anisotropic square-well potential. ${ }^{94}$ The free energy of the nematic phase of our attractive hard-spherocylinder particles can be expressed in algebraic form in the terms of 
the OTF parameter $\alpha$ :

$$
\begin{aligned}
\frac{A^{\mathrm{nem}}(\alpha)}{N k T} & =\frac{A_{\mathrm{iso}}^{\mathrm{id}}}{N k T}+\ln \alpha-1 \\
& +\frac{G(\eta)}{V_{\mathrm{m}}}\left[C_{0}+C_{1} \sqrt{\pi}\left(\frac{1}{\alpha^{1 / 2}}-\frac{15}{16 \alpha^{3 / 2}}\right)\right] \\
& -\frac{\rho \epsilon_{0}}{2 k T}\left[\frac{4 \pi}{3} \lambda^{3} D^{3}-C_{0}-C_{1} \sqrt{\pi}\left(\frac{1}{\alpha^{1 / 2}}-\frac{15}{16 \alpha^{3 / 2}}\right)\right] \\
& -\frac{\rho \epsilon_{2}}{2 k T}\left[\left(\frac{4 \pi}{3} \lambda^{3} D^{3}-C_{0}\right)\left(1-6 \alpha^{-1}\right)\right. \\
& \left.-C_{1} \sqrt{\pi}\left(\alpha^{-1 / 2}-\frac{159}{16} \alpha^{-3 / 2}\right)\right]
\end{aligned}
$$

where for hard spherocylinders the molecular volume is $V_{\mathrm{m}}=\pi D^{3} / 6+\pi L D^{2} / 4$.

The equilibrium value of $\alpha$ can be determined by minimising the total Helmholtz free energy of the system. On differentiating the free energy with respect to $\alpha$, one can factor out a simple cubic equation of $x=\sqrt{\alpha}$ :

$$
\frac{\partial}{\partial \alpha} \frac{A(\alpha)}{N k T}=\frac{1}{x^{5}}\left(x^{3}+a_{2} x^{2}+a_{1} x+a_{0}\right)=0,
$$

where the coefficients depend on the repulsive and attractive intermolecular parameters and the system density through

$$
\begin{aligned}
& a_{0}=\frac{45}{32} C_{1} \sqrt{\pi}\left[\frac{G(\eta)}{V_{\mathrm{m}}}+\frac{\rho \epsilon_{0}}{2 k T}\left(1+\frac{53}{5} \frac{\epsilon_{2}}{\epsilon_{0}}\right)\right] \\
& a_{1}=6 \frac{\rho \epsilon_{2}}{2 k T}\left(C_{0}-\frac{4 \pi}{3} \lambda^{3} D^{3}\right) \\
& a_{2}=-\frac{C_{1} \sqrt{\pi}}{2}\left[\frac{G(\eta)}{V_{\mathrm{m}}}+\frac{\rho \epsilon_{0}}{2 k T}\left(1+\frac{\epsilon_{2}}{\epsilon_{0}}\right)\right] .
\end{aligned}
$$

The solution for $\alpha$ in Equation (17) can be expressed as ${ }^{48,94}$

$$
\begin{aligned}
\alpha_{\mathrm{eq}} & =\frac{1}{9}\left\{a_{2}-2 \sqrt{a_{2}^{2}-3 a_{1}} \cos \left(\frac{2 j \pi}{3}\right.\right. \\
& \left.\left.+\frac{1}{3} \arccos \frac{-27\left[a_{0}-\frac{1}{3} a_{1} a_{2}+\frac{2}{27} a_{2}^{3}\right]}{2\left[a_{2}^{2}-3 a_{1}\right]^{3 / 2}}\right)\right\}^{2}, \quad j=\{0,1,2\} .
\end{aligned}
$$


The equilibrium value of $\alpha_{\mathrm{eq}}$ describing the orientationally ordered nematic phase corresponds to the largest root $(j=0)$.

An important quantity that characterizes the degree of orientational order is the nematic order parameter $S_{2}$, which is usually defined as the orientational average of second Legendre polynomial $P_{2}(\cos (\theta))$ :

$$
S_{2}=\int P_{2}(\cos (\theta)) f(\theta) \mathrm{d} \vec{\omega} .
$$

As the OTF is used to represent the orientational distribution in nematic phase, $f(\theta)=$ $f_{\text {OтF }}(\vec{\omega})$, one can express the nematic order parameter as a function of equilibrium orientational parameter $\alpha_{\text {eq }}:{ }^{48}$

$$
S_{2}=1-\frac{3 \operatorname{coth} \alpha_{\mathrm{eq}}}{\alpha_{\mathrm{eq}}}+\frac{3}{\alpha_{\mathrm{eq}}^{2}} .
$$

In the case of an isotropic state (characterized by $S_{2}=0$ ), the solution becomes much simpler since the orientational distribution is the constant, $f(\vec{\omega})=1 / 4 \pi$. The free energy of the isotropic liquid can then be written as

$$
\begin{aligned}
\frac{A^{\text {iso }}}{N k T} & =\frac{A_{\text {iso }}^{\text {id }}}{N k T}+\frac{G(\eta)}{V_{\mathrm{m}}}\left(C_{0}+\frac{\pi}{4} C_{1}\right) \\
& -\frac{\rho \epsilon_{0}}{2 k T}\left[\frac{4 \pi}{3} \lambda^{3} D^{3}-C_{0}+\frac{\pi}{4} C_{1}\left(\frac{\epsilon_{2}}{8 \epsilon_{0}}-1\right)\right] .
\end{aligned}
$$

The standard thermodynamic relations $A=N \mu-P V$ and $P=-(\partial A / \partial V)_{T}$ allow algebraic expressions for chemical potential $\mu$ and compressibility factor (pressure) $Z=$ $P V / N k T$ in both the isotropic and nematic phases to be obtained. The solution of phase equilibria is determined by equating the chemical potential and pressure of both phases. In the case of our attractive hard spherocylinders, four possible pairs of coexisting fluid phases can be expected: vapour-liquid, vapour-nematic, liquid-nematic and nematic-nematic. There will also be a corresponding three-phase equilibrium coexistence point (e.g., vapour-liquidnematic or vapour-nematic-nematic). The possible types of phase behaviour exhibited by this simple system are examined in the following sections. 


\section{Phase behaviour of attractive hard spherocylinders}

In the present study, hard spherocylinders with an aspect ratio of $L / D$ are enveloped by a square-well of fixed range $\lambda=L / D+1$. The calculated phase diagrams are first represented in terms of dimensionless variables: $T^{*}=k T / \epsilon_{0}$ for the temperature; $P^{*}=P V_{\mathrm{m}} / \epsilon_{0}$ for the pressure; and $\eta=\rho V_{\mathrm{m}}$ for the packing fraction.

The phase behaviour of hard spherocylinders with an isotropic (spherically symmetrical) SW potential, i.e., with $\epsilon_{0} \neq 0$ and $\epsilon_{2}=0$, is presented in Figure 2. The richness of the phase behaviour exhibited by an attractive rod of varying length is consistent with the idea that hard-core interactions are to a great extent responsible for the stabilization of the nematic phase. A detailed discussion of systems of short attractive rods $(L / D<50)$ can be found elsewhere. ${ }^{94}$ Relatively large aspect ratios can be found experimentally in the systems of rod-like colloidal suspensions. ${ }^{11}$ The focus of our current work is on rodlike particles with aspect ratios of $L / D=50,100$ and 150 . One of most striking features of the phase behaviour is the appearance of a region of nematic-nematic phase separation for high molecular elongations. ${ }^{94,96,117-119}$ This behaviour is a consequence of the isotropicnematic transition moving to progressively lower densities as the molecular aspect ratio is increased. The nematic-nematic region involves the coexistence of a low-density (vapourlike) anisotropic state of lower orientational order with a high-density (liquid-like) anisotropic state of higher orientational order, bounded by a critical point at higher temperatures; the order parameters that can be used to distinguish the two nematic phases are therefore the density and the nematic order. For particles of intermediate length the vapour-liquid transition becomes metastable relative to the isotropic fluid-nematic transition; in such a case we refer to the low-density fluid state as an isotropic liquid to allow one to distinguish the two types of phase behaviour.

The effect of including a positive anisotropic attractive contribution $\left(\epsilon_{2}>0\right)$ on the

phase behaviour of our AHSC with $L / D=50$ is presented in Figure 3. Although the weak contribution from the anisotropic attraction does not qualitatively change the type of 

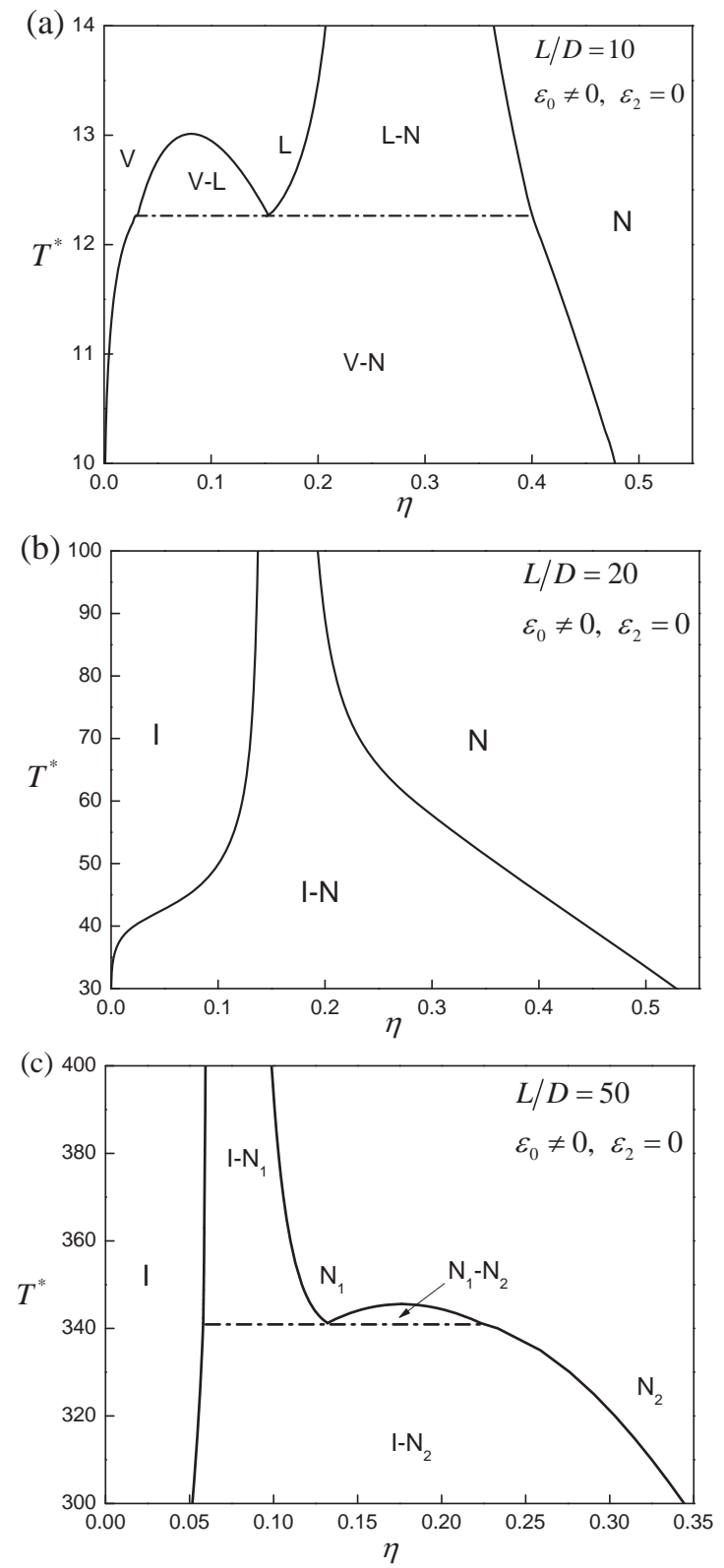

Figure 2: Temperature-density $\left(T^{*}=k T / \epsilon_{0}, \eta=\rho V_{\mathrm{m}}\right)$ representation of the fluid-phase behaviour of attractive hard spherocylinders (AHSCs) with aspect ratios (a) $L / D=10$, (b) $L / D=20$ and (c) $L / D=50$. The rod-like molecules are modelled as hard cylinders of length $L$ and diameter $D$, capped by hard hemispheres of diameter $D$, enveloped by an attractive square well of depth $-\left(\epsilon_{0}+\epsilon_{2} P_{2}(\cos \gamma)\right)$ and range $\lambda=L / D+1$; the temperature is The stable regions are indicated as vapour (V), liquid (L), isotropic fluid (I), and nematic $(\mathrm{N})$. In the case of $L / D=50, \mathrm{~N}_{1}$ and $\mathrm{N}_{2}$ correspond to the low-density and high-density nematic phases, respectively. The dot-dashed lines are the corresponding three phase lines. 


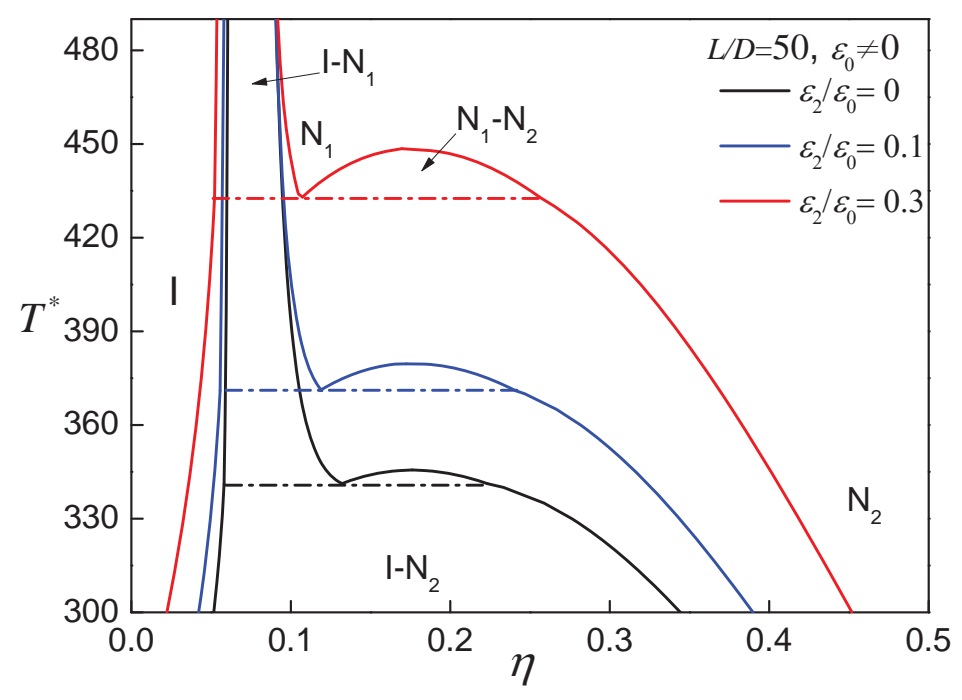

Figure 3: Temperature-density $\left(T^{*}=k T / \epsilon_{0}, \eta=\rho V_{\mathrm{m}}\right)$ representation of the fluid-phase behaviour of attractive hard spherocylinders (AHSCs) with an aspect ratio of $L / D=50$ and positive anisotropic interactions $\epsilon_{2}>0$. See the caption of Figure 2 for further details.

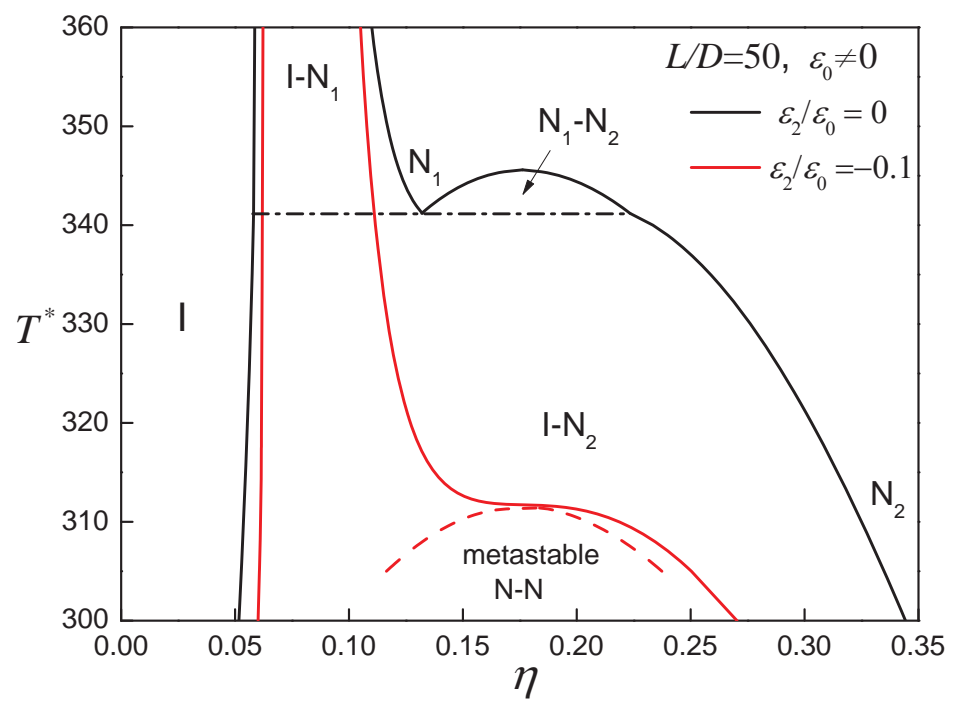

Figure 4: Temperature-density $\left(T^{*}=k T / \epsilon_{0}, \eta=\rho V_{\mathrm{m}}\right)$ representation of the fluid-phase behaviour of attractive hard spherocylinders (AHSCs) with an aspect ratio $L / D=50$ and a negative anisotropic interaction $\epsilon_{2}<0$. The dashed curve represents the metastable nematic-nematic coexistence. See the caption of Figure 2 for further details. 
phase behaviour of the system, the nematic-nematic transition is stabilized on increasing this anisotropic interaction as the latter leads to more favourable parallel relative orientations of the particles. On the other hand, a negative anisotropic attractive contribution to the potential $\left(\epsilon_{2}<0\right)$ destabilizes the nematic-nematic coexistence, as in this case perpendicular relative configurations of the particles are favoured, tending to reduce the effective attractive interactions in the anisotropic state. Since the region of nematic-nematic equilibrium is small, a weak negative anisotropic attraction $\left(\epsilon_{2} / \epsilon_{0}=-0.1\right)$ is sufficient to cause the nematicnematic coexistence to become metastable with respect to the isotropic-nematic transition, as shown in Figure 4. Examples of interactions which would promote perpendicular orientations would be for molecules with large quadrupole moments, such as those characterizing certain aromatic moieties.

The corresponding fluid-phase diagrams of AHSC particles with aspect ratios of $L / D=$ 100 and $L / D=150$ are presented in Figure 5, where the effect of adding both positive and negative anisotropic interactions is examined. The enhanced anisotropic attractive interaction not only promotes the coexistence between two nematic phases but also leads to an increase in the isotropic-nematic-nematic $\left(\mathrm{I}_{-} \mathrm{N}_{1}-\mathrm{N}_{2}\right)$ triple point temperature. The coexistence between the isotropic and the low-density nematic phases $\left(\mathrm{N}_{1}\right)$ is not affected by incorporation of anisotropy in the attractive interactions. As has already been mentioned the position of the isotropic-nematic transition is determined principally by the anisotropy of the rod-like molecular cores, with the coexistence shifting to lower densities as the aspect ratio is increased.

\section{Phase behaviour of solutions of PBLG in DMF}

In order to assess the capabilities of our van der Waals-Onsager approach in describing thermotropic liquid-crystal behaviour, the attractive hard-spherocylinder model is used to represent the PBLG-DMF system. It has been shown experimentally ${ }^{34,39}$ that under certain 

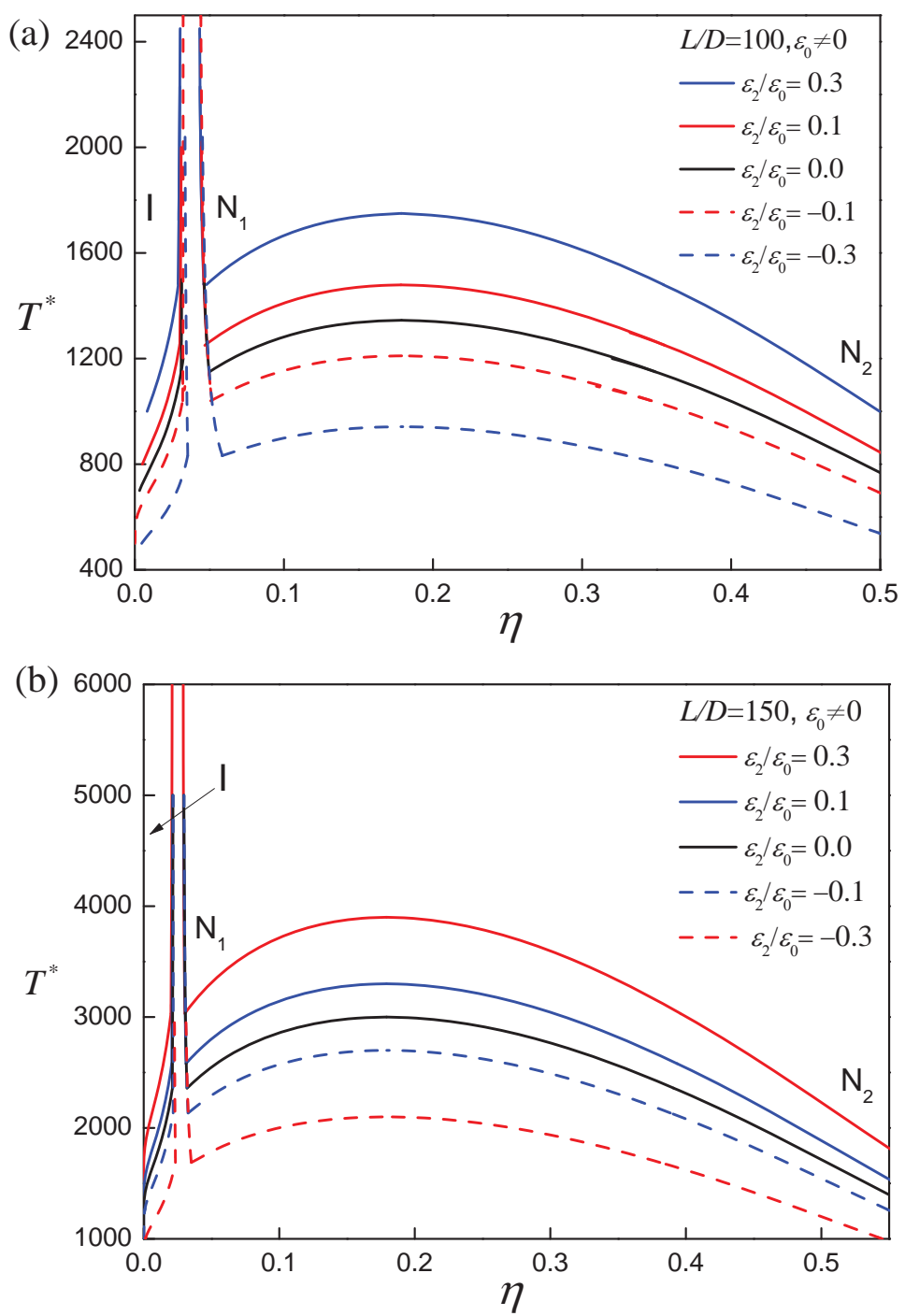

Figure 5: Temperature-density $\left(T^{*}=k T / \epsilon_{0}, \eta=\rho V_{\mathrm{m}}\right)$ representation of the fluid-phase behaviour of attractive hard spherocylinders (AHSCs) with aspect ratios of (a) $L / D=100$ and (b) $L / D=150$ and a varying anisotropic interaction $\epsilon_{2}$. See the caption of Figure 2 for further details. 
conditions the polypeptide PBLG adopts an $\alpha$-helix secondary structure in solutions of DMF; in this conformation the PBLG molecules exhibit a liquid-crystalline phase behaviour which is consistent with that of rod-like particles. ${ }^{20-40}$ The key experimental data for the ordering phase behavior of PBLG solutions in DMF was reported by Miller and co-workers ${ }^{22,23,26-28}$ based on a variety of techniques including proton nuclear magnetic resonance (nmr), polarizing microscopy, differential scanning calorimetry (DSC), and viscometry. The location of the transition between the isotropic phase and the nematic phase found at low concentrations of PBLG is not problematic. It is, however, more difficult to obtain a reliable estimate of the phase boundaries as the concentration is increased due to the high viscosity of the concentrated solutions and the onset of gelation characterized by the appearance of small semi-crystalline aggregates. ${ }^{31,32}$ An inferred phase diagram demarcating the boundary of the homogenous liquid crystalline phase and the coexistence region between two anisotropic phases with different concentrations of PBLG was nevertheless proposed by Miller and coworkers, first based on the splitting of the nmr peaks of DMF on crossing the boundary ${ }^{22}$ and then confirmed by subsequent data from heating/cooling DSC studies ${ }^{23}$ and the temperature/concentration dependence of the cholesteric pitch which was found to exhibit a characteristic kink. ${ }^{27}$ Though gels and crystalline phases are certainly to be expected at the higher concentrations ${ }^{39}$ we will not consider these more complicated features of the phase behaviour of the system in our current study.

The lattice theory of Flory ${ }^{117,120}$ has been employed to explain the phase behaviour of the PBLG-DMF system and several qualitative similarities between the theoretical description and experiment were highlighted. ${ }^{22,23}$ Because of the discrete treatment of the medium in this type of lattice treatment and the artificial sectioning of the rod-like particles, it becomes difficult to make a direct comparison between the lattice model and the real macromolecule. By contrast, the attractive hard-spherocylinder model introduced in our current work is an off-lattice (continuous) model that allows one to relate, in a coarse-grained fashion, the dimensions of the model with that of the rod-like PBLG molecules ( $c f$. Figure 6). 


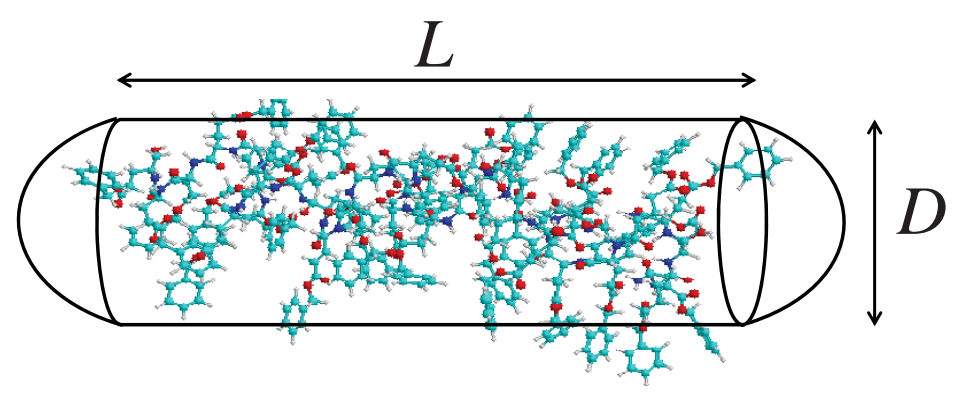

Figure 6: An attractive hard spherocylinder is used to represent the hard-core backbone of the PBLG molecule in its extended $\alpha$-helical conformation. The length of rod $L$ is considered to be temperature dependent to account for the flexibility and conformational changes of the polypeptide in DMF.

There are two crucial features that need to be considered carefully when modelling the PBLG-DMF system: how the conformations of PBLG molecules change over the temperature and density range of interest; and how the solvent (DMF) modulates the effective interactions between the PBLG molecules. Since our model is based on a hard rod there is no explicit account of flexibility that would enable us to incorporate the chain conformation of the macromolecule directly. The characteristic diameter $D_{\mathrm{PBLG}}$ of the PBLG rod is fixed as 14.2 $\AA$ in an attempt to map the extended low-temperature conformation, ${ }^{39}$ which is found to lie between the diameter corresponding to the minimum intermolecular distance (13.0 $\AA$ ) and the diameter of the backbone with extended side chains $(15.2 \AA)$. We allow the rod length to vary with temperature, reflecting the state dependence of the conformations of PBLG. Alternatively the volume of the PBLG model can be fixed so that both the length and the diameter of the rod vary with temperature; we will show that this choice is not found to provide as good a representation of the overall fluid-phase equilibria. The PBLG-DMF mixture is treated as an equivalent single component system, whereby any solvent effects are subsumed into an effective coarse-grained PBLG intermolecular attractive interaction (which now takes on the meaning of a potential of mean force).

A simple conversion is used to interpret the packing fraction $\eta_{\mathrm{PBLG}}$ of the rod-like polymer in terms of its volume fraction $\nu_{\mathrm{PBLG}}$ (which is the experimentally relevant variable). The 
expression for volume fraction of PBLG can be obtained from

$$
\nu_{\mathrm{PBLG}}=\frac{\eta_{\mathrm{PBLG}} M_{\mathrm{w}}}{N_{\mathrm{A}} \rho_{\mathrm{PBLG}} V_{\mathrm{m}, \mathrm{PBLG}}}
$$

where $N_{\mathrm{A}}$ is the Avogadro number and we assume an ideal (additive) volume of mixing for the PBLG-DMF system. In our simple model the molecular volume of PBLG depends on its aspect ratio through: $V_{\mathrm{m}, \mathrm{PBLG}}=\pi D_{\mathrm{PBLG}}^{3} / 6+\pi L_{\mathrm{PBLG}} D_{\mathrm{PBLG}}^{2} / 4$. Because the molecular weight $M_{\mathrm{w}}$ and density $\rho_{\mathrm{PBLG}}$ of PBLG depends on the polymerization conditions, ${ }^{121,122}$ an average prototypical molecular weight of $M_{\mathrm{w}}=310,000 \mathrm{~g} \mathrm{~mol}^{-1}$ is assumed, and a value of $\rho_{\mathrm{PBLG}}=1.283 \mathrm{~g} \mathrm{~cm}^{-3}$ is taken for its density. ${ }^{39}$

There is experimental evidence ${ }^{24,27,36,37,40}$ that solutions of PBLG in some solvents (e.g., DMF or dichloromethane) exhibit a cholesteric (chiral nematic) phase rather than a nematic phase. As the difference in free energy between the two states is very small (owing to the large chiral pitches characteristic of the system), the thermodynamic properties of the cholesteric phase can be approximated by those of the nematic phase. The repulsive interactions between the various conformations of the polypeptide rods are taken into account with a simple hardspherocylindrical core of variable aspect ratio. The effective attractive interactions between helical rods can be described with an enveloping isotropic SW potential, ${ }^{92,93}$ where the depth of the well $\epsilon_{0}$ is tuned to take into account the effect of the solvent medium (and its dielectric properties). In this sense our model is similar to the DLVO potential commonly employed to describe the interactions between colloidal particles in solution. ${ }^{70}$

We have already shown that for long rods $(L / D=100$ and 150$)$ the densities of the isotropic-nematic transition are not affected to a significant degree by the anisotropic contribution (characterized by $\epsilon_{2}$ ) to the attractive intermolecular potential (see Figure 5). Furthermore the liquid-crystalline states of the PBLG system possess a high degree of orientational order so that only near-parallel molecular configurations will contribute to the properties of the system; as a consequence the anisotropy of the attractions can be disregarded. In order to reduce the number of adjustable parameters we have therefore opted to include only an isotropic SW attraction of strength $\epsilon_{0}$ to describe the effective interaction 
between the PBLG molecules in solution. In summary, there are two adjustable parameters: the aspect ratio $L_{\mathrm{PBLG}}^{*}=L_{\mathrm{PBLG}} / D_{\mathrm{PBLG}}$ of the repulsive core and the depth $\epsilon_{0}$ of the enveloping attractive SW potential. At each temperature, starting from the lowest available ( $260 \mathrm{~K}$ ) at which one would expect the most extended PBLG conformation, the values of these two parameters are estimated from the experimental data for the phase boundaries of the two regions of isotropic-nematic coexistence (denoted as $\mathrm{I}^{-\mathrm{N}_{1}}$ and $\mathrm{I}-\mathrm{N}_{2}$ in Figure 7). Once the values of $L_{\mathrm{PBLG}}^{*}(T)$ and $\epsilon_{0}(T)$ are determined one can predict the nematic-nematic phase equilibrium of the PBLG solution as can be seen from Figure 7.

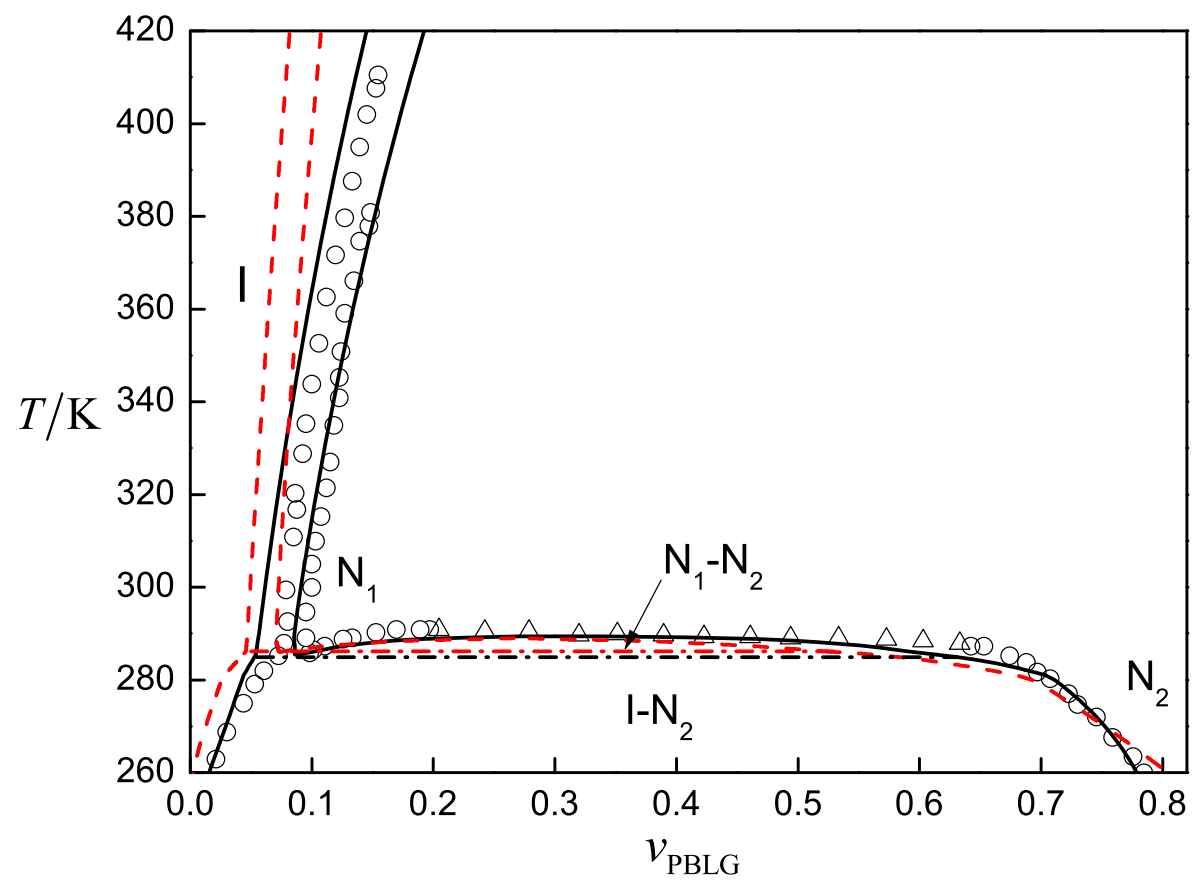

Figure 7: The temperature-volume fraction representation of the phase diagram of solutions of the polypeptide PBLG (molecular weight $M_{\mathrm{w}}=310,000 \mathrm{~g} \mathrm{~mol}^{-1}$ ) in DMF. The symbols represent the experimental data of Miller and co-workers; ${ }^{22,23,27,28}$ in order to differentiate the phase boundary inferred in the high-viscosity region the corresponding data is represented as triangles. The continuous curves correspond to the theoretical description obtained using the van der Waals-Onsager free-energy functional with the attractive hard-spherocylinder (AHSC) model. The two regions of isotropic-nematic coexistence are denoted by $\mathrm{I}_{-} \mathrm{N}_{1}$ and I- $\mathrm{N}_{2}$, the region of nematic-nematic coexistence by $\mathrm{N}_{1}-\mathrm{N}_{2}$, and the dot-dashed line denotes the I- $\mathrm{N}_{1}-\mathrm{N}_{2}$ three-phase coexistence. The description obtained assuming the volume of the PBLG rod is constant is represented with the dashed curves. 
The description of the experimental data $22,23,27,28$ with our algebraic van der WaalsOnsager equation of state is seen to be relatively good. At the temperature of $T=280 \mathrm{~K}$, a low-density isotropic phase $\left(\nu_{\mathrm{PBLG}, \mathrm{I}}=0.0424\right)$ coexists with a concentrated nematic phase $\left(\nu_{\mathrm{PBLG}, \mathrm{N}_{2}}=0.711\right)$. The AHSC model successfully reproduces the fluid-phase behaviour over the entire temperature range. Isotropic-nematic-nematic three-phase coexistence is predicted at a temperature of $T=285.40 \mathrm{~K}$ where an isotropic liquid phase, a low-concentration nematic phase and a high-concentration nematic phase simultaneously coexist. The most salient feature in the phase diagram of the PBLG-DMF system is the equilibrium between low-concentration $\left(\mathrm{N}_{1}\right)$ and high-concentration $\left(\mathrm{N}_{2}\right)$ nematic phases. At a temperature of $T=290 \mathrm{~K}$ the isotropic liquid state $\left(\nu_{\mathrm{PBLG}, \mathrm{I}}=0.0564\right)$ coexists with a low-concentration nematic state $\left(\nu_{\mathrm{PBLG}, \mathrm{N}_{1}}=0.0856\right)$. Above the triple temperature, only a weak temperature dependence of the coexistence concentrations of the isotropic-nematic boundary is found. It is also clear from Figure 7 that the corresponding description of the fluid-phase behaviour obtained by assuming that the volume of the PBLG rod remains constant (corresponding to that of the extended conformation) is not as satisfactory; we therefore do not pursue this assumption any further. It has recently been shown ${ }^{123}$ that the effective volume of model helical hard-core particles increases as they become more elongated (less twisted); this is consistent with our assumption that the effective volume of the PBLG macromolecule is larger (corresponding to a larger aspect ratio) in the more extended low-temperature states. The coexistence between the two nematic phases in solutions of PBLG in DMF is well documented experimentally: ${ }^{20,22,23,27,28,33,36,40}$ the region of nematic-nematic coexistence is bounded between the isotropic-nematic-nematic triple point and the nematic-nematic critical temperature as can be seen clearly in the expanded scale of Figure 8.

The temperature-dependence of the nematic order parameter $S_{2}$ is represented in Figure 9: while the degree of orientational order of the high-concentration nematic state $\mathrm{N}_{2}$, is very high and rather invariant with temperature, that of the low-concentration nematic phase $\mathrm{N}_{1}$ is a monotonically increasing function of temperature. The volume fraction of the $\mathrm{N}_{2}$ state at 


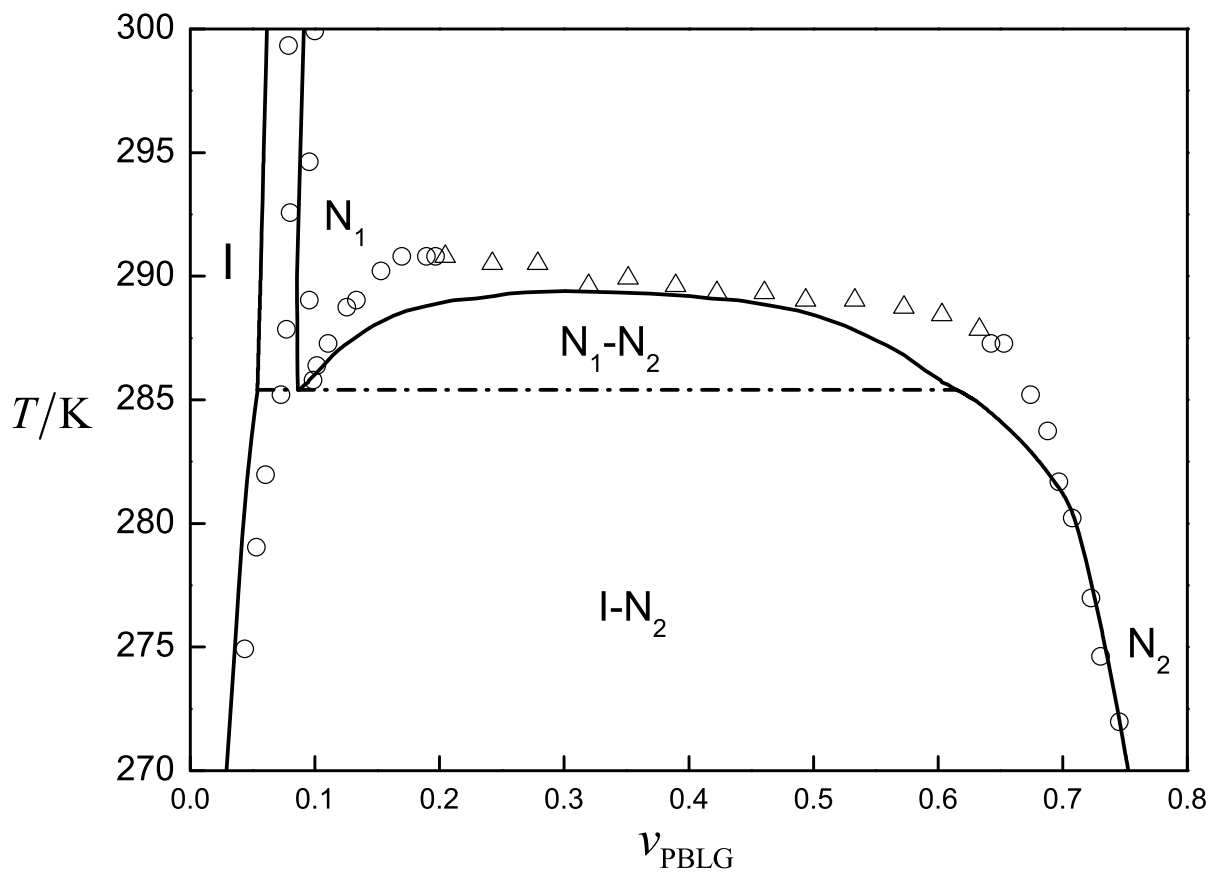

Figure 8: The phase diagram of Figure 7 highlighting the regions of nematic-nematic and isotropic-nematic-nematic three-phase coexistence (see the caption of Figure 7 for further details). 
the I- $\mathrm{N}_{2}$ coexistence boundary ic characterized by an effective packing fraction of $\eta_{\mathrm{N}_{2}} \sim 0.35$ (volume fraction $\nu_{\mathrm{PBLG}, \mathrm{N}_{2}} \sim 0.61$ ). Considering that the aspect ratio of the PBLG rod is $\sim 100$ this corresponds to a relatively dense state, and hence to a high degree of orientation order. The orientational order in the low-concentration nematic phase is seen to be slightly lower.

As mentioned earlier, Miller and co-workers ${ }^{22,23,27,28}$ were the first to report the existence of two coexisting liquid-crystalline states in solutions of PBLG in DMF, though the highconcentration anisotropic states are very difficult to access experimentally because of the high viscosity of the solutions. The existence of two anisotropic states in equilibrium was also confirmed when two different LC textures with the characteristic fingerprint pattern of chiral nematic (cholesteric) phases were observed under a polarizing microscope: ${ }^{25,33,36}$ a high-concentration phase of relatively low pitch was found to coexist with a low-concentration phase of higher pitch. Several factors such as the molecular weight, the nature of the solvent, and the heating and cooling rates affect the phase behaviour observed in the system. With our current coarse-grained model we do not consider the cholesteric ordering of the PBLG macromolecules, assuming instead that the free energy of the system is essentially that of a nematic phase. In Figure 9, the less ordered nematic phase corresponds to the high-pitch cholesteric phase while the highly ordered nematic state represents the low-pitch PBLG-rich state.

The evolution of the orientational order parameter of the nematic phase in coexistence with the isotropic phase is shown in Figure 10. The order parameter decreases with increasing temperature along both the I- $\mathrm{N}_{1}$ and $\mathrm{I}_{-} \mathrm{N}_{2}$ boundaries as the anisotropic phases become less ordered, particularly in the low-concentration $\mathrm{N}_{1}$ phase; a sudden drop can be seen near the isotropic-nematic-nematic I- $\mathrm{N}_{1}-\mathrm{N}_{2}$ triple-point temperature.

The temperature dependence obtained for the anisotropic square-well depth $\epsilon_{0}$ of our AHSC model of PBLG is depicted in Figure 11. The change in the attractive parameter is seen to be quite small over the entire temperature range $\left(\Delta \epsilon_{0} / k_{\mathrm{B}} \sim 0.04 \mathrm{~K}\right)$, and is found 


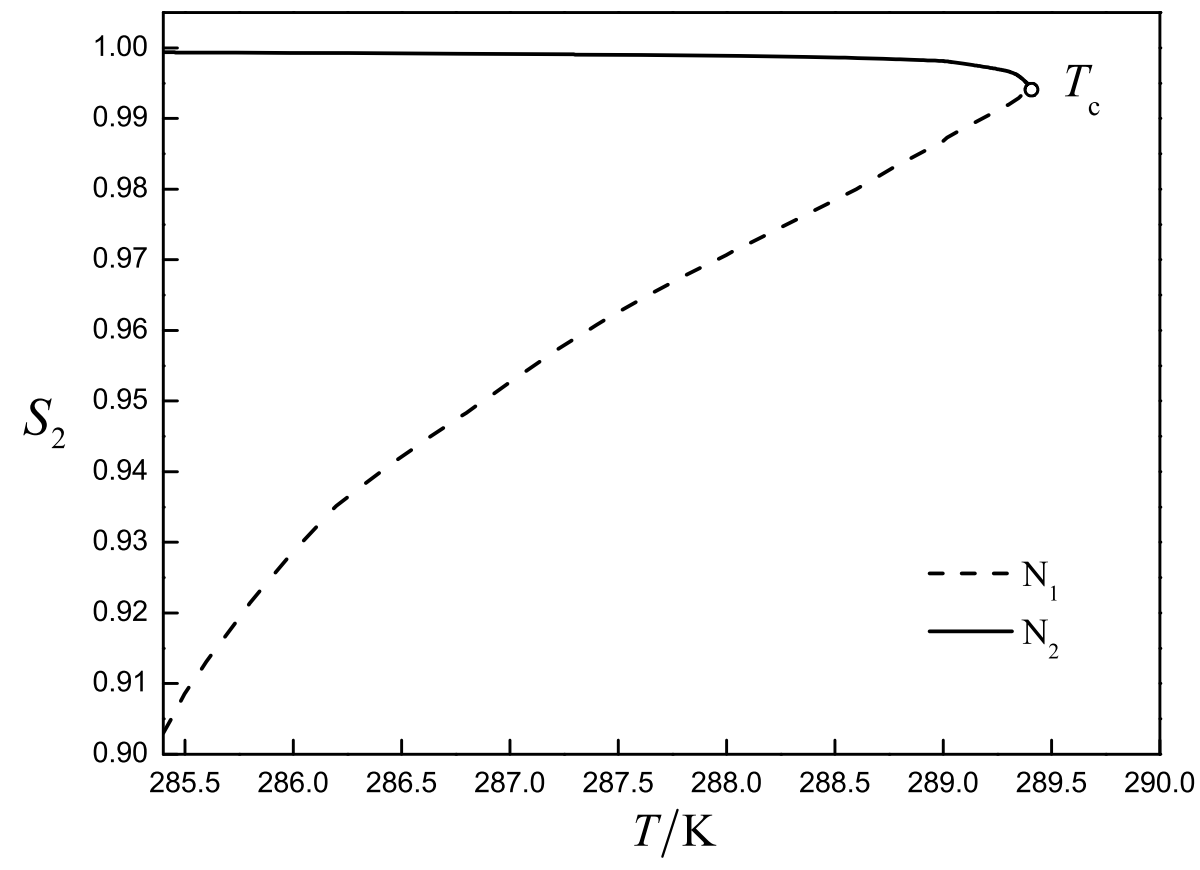

Figure 9: The temperature dependence of the nematic order parameters $S_{2}$ in the coexisting nematic phases of solutions of PBLG (molecular weight $M_{\mathrm{w}}=310,000 \mathrm{~g} \mathrm{~mol}^{-1}$ ) in DMF predicted using the van der Waals - Onsager approach (continuous and dashed curves) with a simple attractive hard-spherocylinder (AHSC) model. The nematic-nematic critical point $T_{\mathrm{c}}$ is also indicated. 
not to affect the phase behaviour of the low-concentration states. In the region below a temperature of $280 \mathrm{~K}$, the energy parameter is $\epsilon_{0}(T<280 \mathrm{~K}) / k_{\mathrm{B}} \sim 0.26 \mathrm{~K}$, while the parameter takes a value of $\epsilon_{0}(T>290 \mathrm{~K}) / k_{\mathrm{B}} \sim 0.22 \mathrm{~K}$ for temperatures above $290 \mathrm{~K}$. In other words, the isotropic-nematic behaviour exhibited by the PBLG-DMF system can essentially be treated as two separate lyotropic liquid-crystalline transitions either side of the triple point; this is not the case for the nematic-nematic coexistence region between the two anisotropic phases. The fact that the effective attractive interactions between the PBLG rods are stronger (cf. the larger values of $\epsilon_{0}$ ) in the high-concentration low-temperature states than in the low-concentration high-temperature states would suggest a fine interplay with the properties of the solvent (e.g., the state dependence of the dielectric constant).

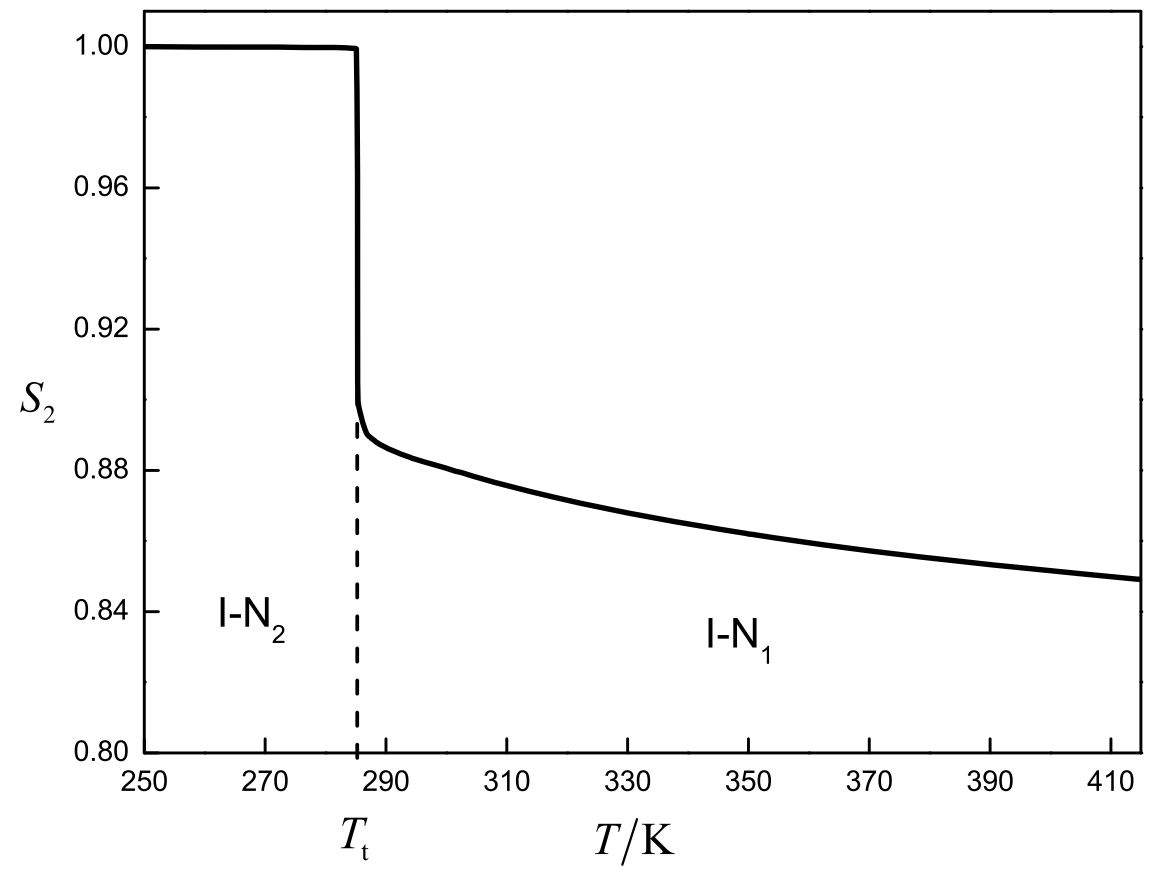

Figure 10: The temperature dependence of the nematic order parameters $S_{2}$ of the anisotropic phase along the isotropic-nematic coexistence boundary predicted using the van der Waals - Onsager approach (continuous curve) with a simple attractive hard-spherocylinder (AHSC) model of solutions of PBLG (molecular weight $M_{\mathrm{w}}=$ 310, $000 \mathrm{~g} \mathrm{~mol}^{-1}$ ) in DMF. The triple temperature $T_{\mathrm{t}}$ is indicated. 


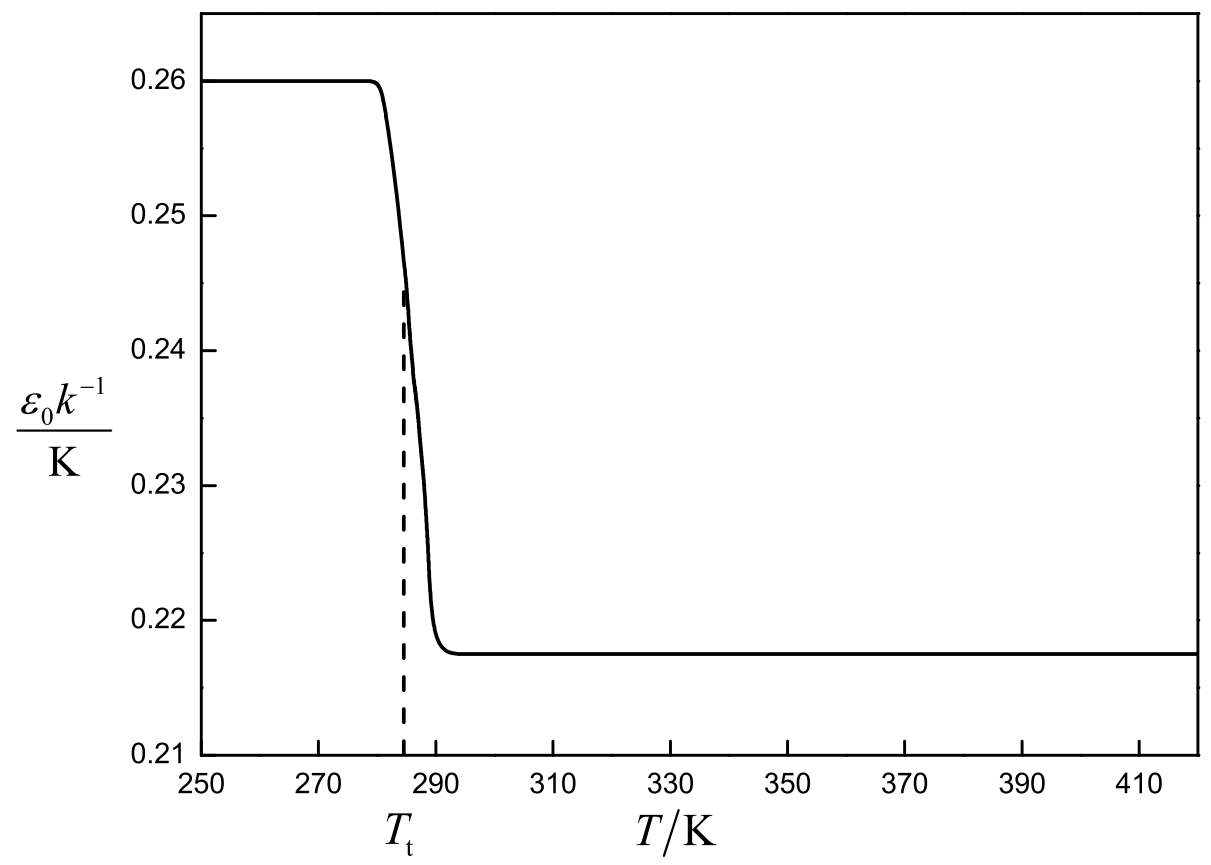

Figure 11: The temperature dependence of isotropic attractive parameter of solutions of PBLG (molecular weight $M_{\mathrm{w}}=310,000 \mathrm{~g} \mathrm{~mol}^{-1}$ ) in DMF estimated using our van der Waals - Onsager approach (continuous curve) with a simple attractive hard-spherocylinder (AHSC) model. The triple temperature $T_{\mathrm{t}}$ is indicated. 
An assessment of the effective aspect ratio obtained for the PBLG rod with our model is now made to ensure the overall shape of the rod is consistent with that of the real PBLG macromolecule. We find that the estimated values of the molecular anisotropy can be described with a simple linear function in the inverse temperature:

$$
L_{\mathrm{PBLG}}^{*}=\frac{L_{\mathrm{PBLG}}}{D_{\mathrm{PBLG}}}=\frac{35003.82}{(T / \mathrm{K})}-21.3179 .
$$

The effective aspect ratio is found to range from $L_{\mathrm{PBLG}}^{*}=62$ to 118 over the temperature range of $420 \mathrm{~K}$ to $260 \mathrm{~K}$. We recall here that the effective diameter of our AHSC model of the PBLG rod is fixed to the value in the extended low-temperature configuration, $D_{\mathrm{PBLG}}=14.2$ $\AA$. The apparent length of the PBLG rod thus varies from $L_{\mathrm{PBLG}} \sim 88 \mathrm{~nm}$ to $168 \mathrm{~nm}$ (see Figure 12), which is in broad agreement with the range of persistence lengths obtained experimentally ${ }^{39}$ from light scattering studies, $L_{\mathrm{PBLG}}^{\exp } \sim 70 \mathrm{~nm}$ and $160 \mathrm{~nm}$; the diameter of $D_{\mathrm{PBLG}}^{\mathrm{exp}} 15.2 \AA$ estimated experimentally ${ }^{39}$ by considering that the side chains of PBLG rod are independent rotators is also in line with the predictions from our AHSC model. When the volume of the rod $V_{\mathrm{m}, \mathrm{PBLG}}$ is assumed to be constant, a range of diameters from $D_{\mathrm{PBLG}}=14.2 \AA$ at $260 \mathrm{~K}$ to $18.0 \AA$ at $420 \mathrm{~K}$ is found to best represent the experimental phase behaviour, corresponding to an average of $16.1 \AA$.

It is important to emphasize that the vapour-liquid and nematic-nematic behaviour exhibited by our rod-like particles is a consequence of the interplay between the inter-particle attractions and the excluded-volume interactions. This is very different to the "vapourliquid" behaviour exhibited by colloidal rod-like particles on addition of non-interacting polymer. ${ }^{124,125}$ In polymer-colloid systems the polymer induces an effective attractive interaction (depletion force) between the colloids (that would otherwise only interact in a purely repulsive fashion) giving rise to a van der Waals-like "vapour-liquid" transition; this equivalent single-component system of attractive colloids can be used to represent the phase behaviour of the mixture ${ }^{126,127}$ The phase diagram exhibited by PBLG in DMF is not a consequence of depletion interactions as the solvent is very small compared to the polypeptide and the behaviour is found to be very sensitive to the temperature of the system. This 


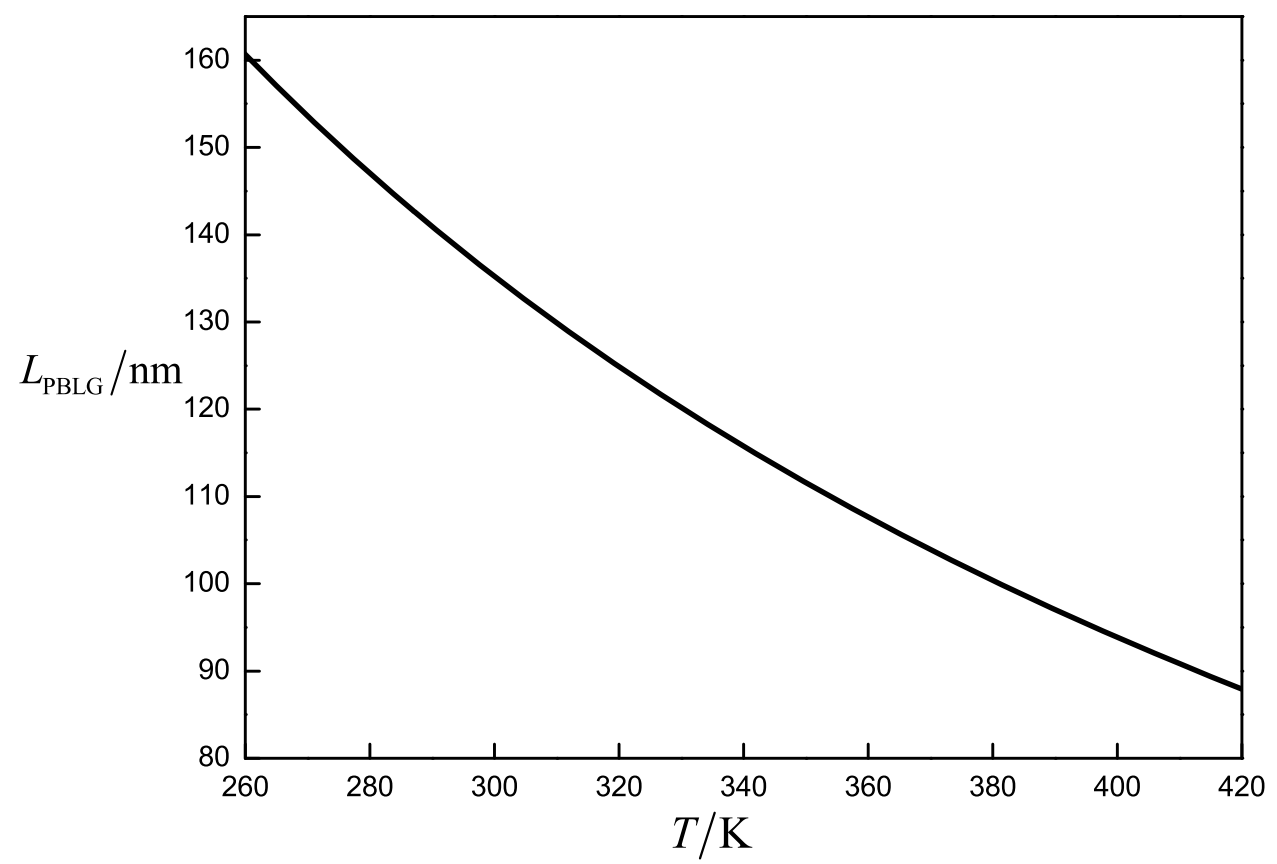

Figure 12: The temperature dependence of the apparent length of the PBLG rod (molecular weight $M_{\mathrm{w}}=310,000 \mathrm{~g} \mathrm{~mol}^{-1}$ ) in DMF estimated using our van der Waals - Onsager approach (continuous curve) with a simple attractive hard-spherocylinder (AHSC) model. 
would suggest that both repulsive (excluded-volume) and attractive interactions between the PBLG molecules are at play.

\section{Conclusions}

In this work, we present a theoretical framework for the description of attractive hardspherocylinder fluids which serve as a basic model for thermotropic rod-like LC systems. The phase behaviour of prolate (spherocylinder) particles are described using a van der Waals - Onsager free-energy functional for the isotropic and nematic states. With the aid of the Onsager trial function to represent the orientation distribution in the nematic phase, the free energy is expressed in algebraic form and the functional variation of free energy then involves the derivative with respect to the Onsager orientational parameter $\alpha$. Using the resulting equation of state, we calculate the phase diagrams of systems of AHSC particles. The effect of the intermolecular parameters (molecular aspect ratio and strength of the isotropic/anisotropic attractive interactions) on the isotropic-nematic phase behaviour of these model attractive rods is examined in detail, and a particular emphasis is placed on the region of nematic-nematic coexistence.

The theory is applied to model the fluid-phase behaviour and orientational order exhibited by solutions of the polypeptide PBLG which form rod-like aggregates in DMF. The PBLG macromolecules are represented as attractive rod-like particles (hard spherocylinders with an enveloping square well) with two temperature-dependent adjustable parameters: the aspect ratio of the hard core and the well-depth of the square well. With our model one is able to faithfully reproduce the regions of isotropic-nematic coexistence as well as the more challenging nematic-nematic phase behaviour exhibited by the PBLG-DMF system.

Though the effective shape of the PBLG rods obtained from our analysis is consistent with the experimental estimates, it should be recognized that our model is rather crude: the molecular flexibility is accounted for in an effective manner, and the attractive sphere 
enveloping the macromolecules is a highly coarse-grained representation of the specific attractive interactions (e.g., surface charges, dipolar interactions, dispersion forces,etc.) present in the system.

An advantage of the perturbative methodology developed here is that it enables one to incorporate other relevant interactions into the model and construct a more realistic representation of the interactions. The Statistical Associating Fluid Theory (SAFT) and its variants, ${ }^{128-131}$ which are based on more detailed information at the united-atom molecular level, have been shown to provide an accurate description of fluid-phase behaviour of complex fluids and fluid mixtures. ${ }^{132,133}$ The SAFT equation of state has recently been employed to develop coarse-grained force fields for direct use in molecular simulation. ${ }^{134-136}$ In future work we plan to take advantage of these developments and improve our model and theoretical approach to provide a predictive platform for the the phase behaviour of thermotropic liquid crystals as well as the structures of the corresponding anisotropic phases.

\section{Acknowledgement}

LW thanks the Department for Business Innovation and Skills, UK and China Scholarship Council for funding a PhD studentship. Funding to the Molecular Systems Engineering Group from the Engineering and Physical Sciences Research Council (EPSRC) of the UK (grants GR/T17595, GR/N35991, EP/E016340, and EP/J014958), the Joint Research Equipment Initiative (JREI) (GR/M94426) is also gratefully acknowledged.

\section{References}

(1) Collings, P. J. Introduction to Liquid Crystals: Chemistry and Physics; Taylor \& Francis, London, 1982.

(2) Chandrasekhar, S. Liquid Crystals, 2nd ed.; Cambridge University Press, Cambridge, 1992. 
(3) de Gennes, P. G.; Prost, J. The Physics of Liquid Crystals, 2nd ed.; Oxford University Press, Oxford, 1993.

(4) Reinitzer, F. Monatshefte für Chemie (Wien) 1888, 9, 421-441.

(5) Livolant, F.; Leforestier, A. Progress in Polymer Science 1996, 21, 1115-1164.

(6) Nakata, M.; Zanchetta, G.; Chapman, B. D.; Jones, C. D.; Cross, J. O.; Pindak, R.; Bellini, T.; Clark, N. A. Science 2007, 318, 1276-1279.

(7) Sato, T.; Teramoto, A. Advances in Polymer Science 1996, 126, 85-161.

(8) Werbowj, R. S.; Gray, D. G. Molecular Crystals and Liquid Crystals 1976, 34, 97-103.

(9) Miller, A. F.; Donald, A. M. Biomacromolecules 2003, 4, 510-517.

(10) Jung, J.-M.; Mezzenga, R. Langmuir 2010, 26, 504-514.

(11) Mezzenga, R.; Jung, J.-M.; Adamcik, J. Langmuir 2010, 26, 10401-10405.

(12) Bawden, F. C.; Pirie, N. W.; Bernal, J. D.; Fankuchen, I. Nature 1936, 138, 10511052.

(13) Parsegian, V. A.; Brenner, S. L. Nature 1976, 259, 632-635.

(14) Dogic, Z.; Fraden, S. Physcial Review Letters 1997, 78, 2417-2420.

(15) Dogic, Z.; Fraden, S. Langmuir 2000, 16, 7820-7824.

(16) Grelet, E.; Fraden, S. Physical Review Letters 2003, 90, 198302.

(17) Purdy, K. R.; Varga, S.; Galindo, A.; Jackson, G.; Fraden, S. Physcial Review Letters 2005, 94, 057801.

(18) Varga, S.; Purdy, K.; Galindo, A.; Fraden, S.; Jackson, G. Physical Review E 2005, 72, 051704 . 
(19) Dogic, Z.; Fraden, S. Current Opinion in Colloid $\mathcal{E}$ Interface Science 2006, 11, 47-55.

(20) Robinson, C. Tetrahedron 1961, 13, 219-234.

(21) Fujita, H.; Teramoto, A.; Okita, K.; Yamashita, T.; Ikeda, S. Biopolymers 1966, 4, 769-779.

(22) Wee, E. L.; Miller, W. G. Journal of Physical Chemistry 1971, 75, 1446-1452.

(23) Miller, W. G.; Wu, C. C.; Wee, E. L.; Santee, G. L.; Rai, J. H.; Goebel, K. G. Pure and Applied Chemistry 1974, 38, 37-58.

(24) DuPré, D. B.; Duke, R. W. Journal of Chemical Physics 1975, 63, 143-148.

(25) Rajan, V. T.; Woo, C. Physical Review A 1980, 21, 990-997.

(26) Zimmel, J. M.; Wu, C. C.; Miller, W. G.; Mason, R. P. Journal of Physical Chemistry 1983, 87, 5435-5443.

(27) Russo, P. S.; Miller, W. G. Macromolecules 1983, 16, 1690-1693.

(28) Russo, P. S.; Miller, W. G. Macromolecules 1984, 17, 1324-1331.

(29) Yamakawa, H. Annual Review of Physical Chemistry 1984, 35, 23-47.

(30) Uematsu, I.; Uematsu, Y. Advances in Polymer Science 1984, 59, 37-73.

(31) Ginzburg, B. M.; Syromyatnikova, T. A.; Frenkel, S. Y. Polymer Bulletin 1985, 13, $139-144$.

(32) Hill, A.; Donald, A. M. Polymer 1988, 29, 1426-1432.

(33) Horton, J. C.; Donald, A. M.; Hill, A. Nature 1990, 346, 44-45.

(34) McMaster, T.; Miles, H.; Morris, V. Journal of Vacuum Science and Technology A 1990, 8, 648-651. 
(35) Tracy, M. A.; Pecora, R. Annual Review of Physical Chemistry 1992, 43, 525-557.

(36) Mori, N.; Itou, S. Chemistry Letters 1994, 2203-2206.

(37) Lin, J.; Abe, A.; Furuya, H.; Okamoto, S. Macromolecules 1996, 29, 2584-2589.

(38) Tipton, D. L.; Russo, P. S. Macromolecules 1996, 29, 7402-7411.

(39) Ginzburg, B. M.; Shepelevskii, A. A. Journal of Macromolecular Science, Part B: Physics 2003, B42, 1-56.

(40) Yen, C.; Edo, S.; Oka, H.; Tokita, M.; Watanabe, J. Macromolecules 2008, 41, 37273733.

(41) Frenkel, D.; Mulder, B. M. Molecular Physics 1985, 55, 1171-1192.

(42) Frenkel, D. Molecular Physics 1987, 60, 1-20.

(43) Frenkel, D.; Lekkerkerker, H. N. W.; Stroobants, A. Nature 1988, 332, 822-823.

(44) Vega, C.; Frenkel, D. Molecular Physics 1989, 67, 633-650.

(45) Samborski, A.; Evans, G. T.; Mason, C. P.; Allen, M. P. Molecular Physics 1994, 81, $263-276$.

(46) McGrother, S. C.; Williamson, D. C.; Jackson, G. Journal of Chemical Physics 1996, $104,6755-6771$.

(47) Bolhuis, P. G.; Frenkel, D. Journal of Chemical Physics 1997, 106, 666-687.

(48) Franco-Melgar, M.; Haslam, A. J.; Jackson, G. Molecular Physics 2008, 106, 649-678.

(49) Vroege, G. J.; Lekkerkerker, H. N. W. Reports on Progress in Physics 1992, 55, 12411309.

(50) Allen, M. P.; Evans, G. T.; Frenkel, D.; Mulder, B. M. Advances in Chemical Physics 1993, 86, 1-166. 
(51) Onsager, L. Physcial Review 1942, 62, 558-559.

(52) Onsager, L. Annals of the New York Academy of Sciences 1949, 51, 627-659.

(53) Parsons, J. D. Physical Review A 1979, 19, 1225-1230.

(54) Lee, S. D. Journal of Chemical Physics 1987, 87, 4972-4974.

(55) Lee, S. D. Journal of Chemical Physics 1988, 89, 7036-7037.

(56) Carnahan, N. F.; Starling, K. E. Journal of Chemical Physics 1969, 51, 635-636.

(57) Esztermann, A.; Reich, H.; Schmidt, M. Physical Review E 2006, 73, 011409.

(58) Hansen-Goos, H.; Mecke, K. Physical Review Letters 2009, 102, 018302.

(59) Cheung, D. L.; Anton, L.; Allen, M. P.; Masters, A. J. Physical Review E 2006, 73, 061204.

(60) Cheung, D. L.; Anton, L.; Allen, M. P.; Masters, A. J.; Phillips, J.; Schmidt, M. Physical Review E 2008, 78, 041201.

(61) Maier, W.; Saupe, A. Zeitschrift für Naturforschung 1958, 13a, 564.

(62) Maier, W.; Saupe, A. Zeitschrift für Naturforschung 1959, 14a, 882.

(63) Chandrasekhar, S.; Krishnamurti, D.; Madhusudana, N. V. Molecular Crystals and Liquid Crystals 1969, 8, 45.

(64) Chandrasekhar, S.; Madhusudana, N. V. Acta Crystallographic 1971, 27, 303.

(65) Humphries, R. L.; James, P. G.; Luckhurst, G. R. Journal of the Chemical Society, Faraday Transactions 2 1972, 68, 1031-1044.

(66) Luckhurst, G. R.; Zannoni, C.; Nordio, P. L.; Segre, U. Molecular Physics 1975, 30, 1345. 
(67) Luckhurst, G. R., Gray, G. W., Eds. The Molecular Physics of Liquid Crystals; Academic Press, London, 1979.

(68) Barker, J. A.; Henderson, D. Review of Modern Physics 1976, 48, 587-671.

(69) Chandler, D.; Weeks, J. D.; Andersen, H. C. Science 1983, 220, 787-794.

(70) Hansen, J.-P.; McDonald, I. R. Theory of Simple Liquids, 3rd ed.; Academic Press, New York, 2006.

(71) Kimura, H. Journal of the Physical Society of Japan 1974, 36, 1280-1287.

(72) Cotter, M. A. Journal of Chemical Physics 1977, 66, 1098-1106.

(73) Cotter, M. A. Journal of Chemical Physics 1977, 66, 4710-4711.

(74) Cotter, M. A. Journal of Chemical Physics 1977, 67, 4268-4270.

(75) Gelbart, W. M.; Baron, B. A. Journal of Chemical Physics 1977, 66, 207-213.

(76) Gelbart, W. M.; Boris, B. Accounts of Chemical Research 1980, 13, 290-296.

(77) Bolhuis, P. G.; Stroobants, A.; Frenkel, D.; Lekkerkerker, H. N. W. Journal of Chemical Physics 1997, 107, 1551-1564.

(78) Williamson, D. C. Molecular Physics 1998, 95, 319-329.

(79) Williamson, D. C.; del Rio, F. Journal of Chemical Physics 1998, 109, 4675-4686.

(80) Williamson, D. C.; Guevara, Y. Journal of Physical Chemistry B 1999, 103, 75227530.

(81) Teixeira, P. I. C. Molecular Physics 1999, 96, 805-811.

(82) Garcia, E.; Williamson, D. C.; Martinez-Richa, A. Molecular Physics 2000, 98, 179192. 
(83) García-Sanchez, E.; Martínez-Richa, A.; Villegas-Gasca, J. A.; Mendoza-Huizar, L. H.; Gil-Villegas, A. Journal of Physical Chemistry A 2002, 106, 10342-10349.

(84) McGrother, S. C.; Gil-Villegas, A.; Jackson, G. Journal of Physics: Condensed Matter 1996, 8, 9649-9655.

(85) Gil-Villegas, A.; McGrother, S. C.; Jackson, G. Chemical Physics Letters 1997, 269, $441-447$.

(86) Groh, B.; Dietrich, S. Physical Review E 1997, 55, 2892-2901.

(87) McGrother, S. C.; Gil-Villegas, A.; Jackson, G. Molecular Physics 1998, 95, 657-673.

(88) Varga, S.; Szalai, I.; Liszi, J.; Jackson, G. Journal of Chemical Physics 2002, 116, 9107-0119.

(89) Williamson, D. C.; Thacker, N. A.; Williams, S. R. Physical Review E 2005, 71, 021702.

(90) Varga, S.; Jackson, G. Chemical Physics Letters 2003, 377, 6-12.

(91) Varga, S.; Jackson, G. Molecular Physics 2006, 104, 3681-3691.

(92) Wensink, H. H.; Jackson, G. Journal of Chemical Physics 2009, 130, 234911.

(93) Wensink, H. H.; Jackson, G. Journal of Physics: Condensed Matter 2011, 23, 194107.

(94) Franco-Melgar, M.; Haslam, A. J.; Jackson, G. Molecular Physics 2009, 107, 23292358.

(95) Stone, A. The Theory of Intermolecular Forces; Clarendon Press, 1996.

(96) Khokhlov, A. R.; Semenov, A. N. Journal of Statistical Physics 1985, 38, 161-182.

(97) Hentschke1990, Macromolecules 1990, 23, 1192-1196. 
(98) Odijk, T. Macromolecules 1986, 19, 2313-2329.

(99) Vroege, G. J.; Odijk, T. Macromolecules 1988, 21, 2848-2858.

(100) Chen, Z. Y. Macromolecules 1993, 26, 3419-3423.

(101) DuPré, D. B.; Yang, S. Journal of Chemical Physics 1991, 94, 7466-7477.

(102) Dijkstra, M.; Frenkel, D. Physical Review E 1995, 51, 5891-5898.

(103) Hino, T.; Prausnitz, J. M. Liquid Crystals 1997, 22, 317-326.

(104) McGrother, S. C.; Sear, R. P.; Jackson, G. Journal of Chemical Physics 1997, 106, $7315-7330$.

(105) Williamson, D. C.; Jackson, G. Journal of Chemical Physics 1998, 108, 10294-10302.

(106) Hino, T.; Prausnitz, J. M. Polymer 1999, 40, 1241-1250.

(107) Wessels, P. P. F.; Mulder, B. M. Journal of Physics: Condensed Matter 2006, 18, 9335-9357.

(108) Jiang, Y.; Chen, J. Z. Y. Macromolecules 2010, 43, 10668-10678.

(109) Zheng, Y. X.; Yu, Y. X.; Li, Y. F. Industrial \& Engineering Chemistry Research 2011, 50, 6460-6469.

(110) Dennison, M.; Dijkstra, M.; van Roij, R. Physical Review Letters 2011, 106, 208302.

(111) Dennison, M.; Dijkstra, M.; van Roij, R. Journal of Chemical Physics 2011, 135, 144106.

(112) Emelyanenko, A. V.; Osipov, M. A.; Dunmur, D. A. Physical Review E 2000, 62, $2340-2352$.

(113) Emelyanenko, A. V. Physical Review E 2003, 67, 031704. 
(114) Zwanzig, R. W. Journal of Chemical Physics 1954, 22, 1420-1426.

(115) Wu, L.; Wensink, H. H.; Jackson, G.; Muller, E. A. Molecular Physics 2012, 110, $1269-1288$.

(116) Wu, L.; ; Jackson, G.; Muller, E. A. International Journal of Molecular Sciences 2013, 14, 16414-16442.

(117) Flory, P. J. Proceedings of the Royal Society of London. Series A, Mathematical and Physical Sciences 1956, 234, 60-73.

(118) Warner, M.; Flory, P. J. Journal of Chemical Physics 1980, 73, 6327-6332.

(119) Varga, S.; C., W. D.; Szalai, I. Molecular Physics 1999, 96, 1695-1703.

(120) Flory, P. J. Proceedings of the Royal Society of London. Series A, Mathematical and Physical Sciences 1956, 234, 73-89.

(121) DeLong, L. M.; Russo, P. S. Macromolecules 1991, 24, 6139-6155.

(122) Inomata, K.; Shimizu, H.; Nose, T. Journal of Polymer Science: Part B: Polymer Physics 2000, 38, 1331-1340.

(123) Frezza, E.; Ferrarini, A.; Kolli, H. B.; Giacometti, A.; Cinacchi, G. Journal of Chemical Physics 2013, 138, 164906.

(124) Warren, P. B. J. Phys. I France 1994, 4, 2.

(125) Sear, R. P.; Jackson, G. Journal of Chemical Physics 1995, 103, 8684-8693.

(126) Paricaud, P.; Varga, S.; Jackson, G. Journal of Chemical Physics 2003, 118, 85258536.

(127) Lekkerkerker, H. N. W.; Tuiner, R. Colloids and the Depletion Interaction; Springer, Heidelberg, 2011. 
(128) Chapman, W. G.; Gubbins, K. E.; Jackson, G.; Radosz, M. Industrial \& Engineering Chemistry Research 1990, 29, 1709-1721.

(129) Gil-Villegas, A.; Galindo, A.; Whitehead, P. J.; Mills, S. J.; Jackson, G.; Burgess, A. N. Journal of Chemical Physics 1997, 106, 4168-4186.

(130) Lymperiadis, A.; Adjiman, C. S.; Galindo, A.; Jackson, G. Journal of Chemical Physics 2007, 127, 234903.

(131) Lymperiadis, A.; Adjiman, C. S.; Jackson, G.; Galindo, A. Fluid Phase Equilibria 2008, 274, 85-104.

(132) Müller, E. A.; Gubbins, K. E. Industrial \& Engineering Chemistry Research 2001, 40, 2193-2211.

(133) McCabe, C.; Galindo, A. In Applied Thermodynamics of Fluids: "SAFT Associating Fluids and Fluid Mixtures"; Goodwin, A. R., Sengers, J., Peter, C. J., Eds.; Royal Society of Chemistry, London, 2010.

(134) Avendaño, C.; Lafitte, T.; Galindo, A.; Adjiman, C. S.; Jackson, G.; Müller, E. A. Journal of Physical Chemistry B 2012, 115, 11154-11169.

(135) Lafitte, T.; Avendaño, C.; Papaioannou, V.; Galindo, A.; Adjiman, C. S.; Jackson, G.; Müller, E. A. Molecular Physics 2012, 110, 1189-1203.

(136) Avendaño, C.; Lafitte, T.; Adjiman, C. S.; Galindo, A.; Müller, E. A.; Jackson, G. Journal of Physical Chemistry B 2013, 117, 2717-2733. 


\section{Graphical TOC Entry}

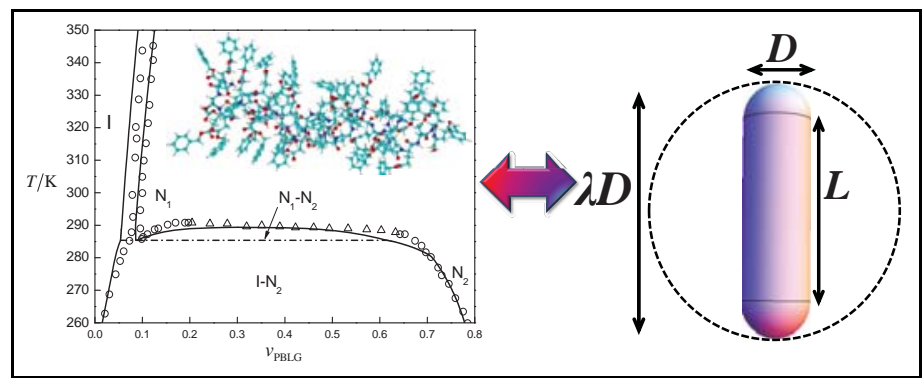

